\title{
RELATING VFCS ON THIN COMPACTIFICATIONS
}

\author{
ELENY-NICOLETA IONEL AND THOMAS H. PARKER
}

\begin{abstract}
Many moduli spaces that occur in geometric analysis admit "Fredholm-stratified thin compactifications" in the sense of [IP1] and hence admit a relative fundamental class (RFC), also as defined in IP1. We extend these results, emphasizing the naturality of the RFC, eliminating the need for a stratification, and proving three compatibility results: the invariants defined by the RFC agree with those defined by pseudo-cycles, the RFC is compatible with cutdown moduli spaces, and the RFC agrees with the virtual fundamental class (VFC) constructed by J. Pardon via implicit atlases in all cases where both are defined.
\end{abstract}

In symplectic Gromov-Witten theory and in gauge theories, the central object of study is a "universal moduli space". It arises by considering the moduli space $\mathcal{M}$ of all pairs $(\varphi, p)$ where $\varphi$ is a solution, modulo gauge, of some family of non-linear elliptic PDEs parameterized by the elements $p$ in a Banach space $\mathcal{P}$. This space $\mathcal{M}$ embeds in one or more (relative) "compactifications" $\overline{\mathcal{M}}$, creating a diagram

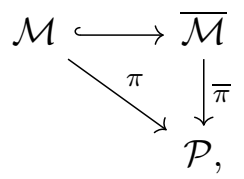

where $\pi$ is differentiable and $\bar{\pi}$ is proper and continuous. This determines an entire category whose objects are pullback diagrams ("proper base changes")

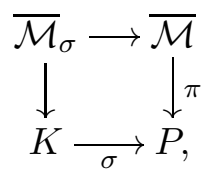

where $\sigma$ is a proper continuous map from a path-connected space. Following [IP1], a relative fundamental class is a functor on this category that associates to each diagram (0.2) a Cech homology class

$$
\left[\overline{\mathcal{M}}_{\sigma}\right]^{r f c} \in \check{\mathrm{H}}_{*}\left(\overline{\mathcal{M}}_{\sigma}\right)
$$

that satisfies a normalization condition. The normalization consists of the requirement that the fiber $\overline{\mathcal{M}}_{p}=\pi^{-1}(p)$ over a dense set of $p \in \mathcal{P}$ is a "thinly compactified manifold" and that, taking $\sigma$ to be the inclusion of $p,\left[\overline{\mathcal{M}}_{p}\right]^{r f c}$ is the fundamental class $\left[\overline{\mathcal{M}}_{p}\right]$. As in Sections 1 and 2, one can use the continuity property of Cech homology to show that there is a unique induced class in the Čech homology of the fiber $\overline{\mathcal{M}}_{q}$ over every point $q$ in $\mathcal{P}$, which is the conventional viewpoint on what a virtual fundamental class should be. Naturality implies that the relative fundamental class is invariant under deformations.

The existence of an RFC was proved in [P1] under the assumption that $\overline{\mathcal{M}}$ is a "Fredholm stratified thin compactification". The first aim of this paper is to strengthen the existence result by replacing the stratification requirement by a covering condition which is much easier to show in applications (see Definition 2.1). 
There are several existing and proposed methods of defining invariants in geometric analysis approaches to Gromov-Witten theory and other gauge theories. Some of these involve constructing a "virtual fundamental class" (VFC) similar to the RFC above. Here, we consider three:

(1) The standard definition using pseudo-cycles [RT1, [MS].

(2) The signed count of elements in a 0-dimensional cutdown moduli space.

(3) The VFC defined by J. Pardon using implicit atlases [Pd].

These vary in the techniques needed for their construction, and in the required assumptions on the space $\overline{\mathcal{M}}$. Only a few results are known about how they are related. The second goal of this paper is to establish an initial "common setting" in which these definitions are comparable and equal.

This paper has four parts and two appendices.

PART I. Sections 1 and 2 review and extend the results of [IP1] on the existence of a relative fundamental class. As in [IP1, the construction involves a combination of Steenrod and Cech homologies, together with the Sard-Smale theorem. The presentation emphasizes the naturality properties, and is nearly self-contained, requiring only the Extension Lemma 3.4 from [P1]. The main result is Theorem 2.3. if a compactified moduli space (0.1) is a "Fredholm thin compactification" as in Definition 2.1, then it admits a unique relative fundamental class. As a result, Definition 2.1 delineates the structure that must be verified in applications.

This approach establishes the existence of an RFC with no need for gluing theorems at the boundary of $\mathcal{M}$, and no need to repeatedly make cobordism arguments, which now follow from the naturality of the RFC.

PART 2. Sections 3-5 relate the numerical invariants (1.23) defined by an RFC to intersections of pseudo-cycles. This is useful because many gauge theory invariants were originally defined using intersections of pseudo-cycles, as was done for the Gromov-Witten invariants of symplectic manifolds in [RT1] and [MS].

Section 3 shows that a pseudo-cycle determines a Steenrod homology class and relates it, under appropriate assumptions, to the pushforward of the fundamental class of a thin compactification (Lemmas 3.3 and 3.6). Section 4 shows that the geometric and homological intersection numbers of pseudo-cycles are equal (Proposition 4.1). Sections 5 moves to the family setting and establishes our first compatibility result. Theorem 5.2 states that, for maps from a Fredholm thin compactification, the pushforward of the RFC is equal in Cech homology to the pseudo-cycle class defined by the images of generic fibers. In particular, the numerical invariants (1.23) defined by the RFC are equal to those defined by the pseudo-cycle approach.

PART 3. In Gromov-Witten and gauge theories, one often uses maps (or sections of bundles) to impose constraints; these determine subsets of the moduli space often called "cutdown" moduli spaces, defined as the inverse image of a submanifold $V$ cf. (6.6). We show that if $f$ is (fully) transverse to $V$, the cutdown family is itself a Fredholm thin compactification, thus carries an RFC.

Algebraic geometers have a formula, involving the cap product with a Thom class, that relates the virtual fundamental classes of the original moduli space and the cutdown space. Section 6 establishes our second compatibility result: Proposition 6.4 shows that the exact same formula applies for our RFC. 
PART 4. Sections 7 and 8 relate our RFC with the virtual fundamental class (VFC) constructed by J. Pardon. Pardon's construction applies to spaces that admit an "implicit atlas" as is defined in $[\mathrm{Pd}]$. In particular, an implicit atlas contains a special chart, called the regular locus $X_{\emptyset}^{\text {reg }}$, which is an oriented manifold. Section 7 proves the fact, communicated

to us by Pardon, that the restriction of the VFC to $X_{\emptyset}^{r e g}$ is the fundamental class of $X_{\emptyset}^{r e g}$. This result - Lemma 7.1 - is independent of the previous sections, and can be viewed as an addendum to Pardon's paper; the proof requires familiarity with $[\mathrm{Pd}]$. It is used in Section 8 to prove our third compatibility result: our RFC agrees with Pardon's VFC for thin families that admit an implicit atlas (Theorem 8.3).

Appendix A summarizes needed facts about Steenrod, Čech and Borel-Moore homologies, including cap products, fundamental classes and intersection pairings. In fact, we mostly work in a context where Steenrod and Borel-Moore homologies are isomorphic, and make use of the well-developed literature on intersection theory for Borel-Moore theory. Appendix B lists results from Dimension theory that are needed in Sections 1-5.

Throughout, all maps are assumed to be continuous, and all spaces are Hausdorff spaces. The term manifold means a Hausdorff topological manifold, with additional properties (e.g. metrizability) only where explicitly stated at beginning of each section. For simplicity, after Section 1 , we use only homology theories with constant coefficients in $R=\mathbb{Z}$ or $\mathbb{Q}$. One can work consistently with a single homology theory by taking $R=\mathbb{Q}$, as we note at the end of Section 1 .

\section{Thin COMPACTIFICATIONS AND FAMILIES}

The notion of a relatively thin family was defined in [IP1, and used to define relative fundamental classes. This section and the next summarize and generalize the relevant definitions, first for a single space $M$, then for families. We refer the reader to Sections 2 and 3 of [IP1] for further details, and to Appendix [A, [Mas], and [ES] for background facts about homology theories.

Steenrod homology ${ }^{s} \mathrm{H}_{*}$ is a homology theory based on infinite chains; it is defined on the category $\mathcal{A}_{L C}$ of locally compact Hausdorff spaces and proper continuous maps as in Mas, Chapter 4]. It satisfies the Eilenberg-Steenrod axioms, including the exactness axiom. It has three additional properties that distinguish it from singular homology and make it especially well-suited for our purposes:

- For each open set $U \subseteq X$ there is a natural "restriction" homomorphism

$$
\rho_{U}:{ }^{s} \mathrm{H}_{*}(X) \rightarrow{ }^{s} \mathrm{H}_{*}(U) .
$$

- For each closed set $\iota: A \hookrightarrow X$, there is a natural long exact sequence

$$
\cdots \longrightarrow{ }^{s} \mathrm{H}_{p}(A) \stackrel{\iota_{*}}{\longrightarrow}{ }^{s} \mathrm{H}_{p}(X) \stackrel{\rho}{\longrightarrow}{ }^{s} \mathrm{H}_{p}(X \backslash A) \stackrel{\partial}{\longrightarrow}{ }^{s} \mathrm{H}_{p-1}(A) \longrightarrow \cdots
$$

- Each oriented $d$-dimensional manifold $M$, whether compact or not, has a fundamental class

$$
[M] \in{ }^{s} \mathrm{H}_{d}(M ; G)
$$

for any abelian coefficient group $G$. Then open subsets of $U \subseteq M$ are manifolds with the induced orientation, and

$$
\rho_{U}[M]=[U] .
$$


1.1. Thin compactifications. Our starting point is the following definition, which was introduced in [P1].

Definition 1.1. A thin compactification of oriented d-dimensional topological manifold $M$ is a compact Hausdorff space $\bar{M}$ containing $M$ such that $S=\bar{M} \backslash M$ is a closed subset of codimension 2 in Steenrod homology:

$$
{ }^{s} \mathrm{H}_{k}(S)=0 \quad \forall k>d-2 .
$$

Under condition (1.4), the exact sequence (1.2) for $(\bar{M}, S)$ shows that restriction to $M$ gives an isomorphism

$$
\rho_{M}:{ }^{s} \mathrm{H}_{d}(\bar{M} ; G) \stackrel{\cong}{\longrightarrow}{ }^{s} \mathrm{H}_{d}(M ; G) .
$$

Hence the fundamental class $[M]$ extends uniquely to a class of $[\bar{M}] \in{ }^{s} \mathrm{H}_{d}(\bar{M} ; G)$ defined by

$$
\rho_{M}[\bar{M}]=[M] .
$$

One advantage of using Steenrod homology is that this extension exists with no assumptions on the differentiability or triangularizability of $\bar{M}$, and without any need to describe the structure of neighborhoods of $\bar{M} \backslash M$ in $\bar{M}$.

Similar considerations apply to cobordisms. A thin compactified cobordism between $\bar{M}_{1}$ and $\bar{M}_{2}$ is a compact Hausdorff pair $(\bar{W}, S)$ such that

(i) $W=\bar{W} \backslash S$ is an oriented cobordism between the $d$-dimensional manifolds $M_{1}$ and $M_{2}$.

(ii) $\bar{M}_{i} \subset \bar{W}$ is a thin compactification of $M_{i}$ for $i=1,2$, and $\bar{M}_{1} \cap \bar{M}_{2}=\emptyset$.

(iii) ${ }^{s} \mathrm{H}_{k}(S)=0$ for all $k \geq d$.

As in [IP1, Corollary 2.12], it follows that

$$
\left(\iota_{1}\right)_{*}\left[\bar{M}_{1}\right]=\left(\iota_{2}\right)_{*}\left[\bar{M}_{2}\right] \quad \text { in } \quad{ }^{s} \mathrm{H}_{d}(\bar{W}),
$$

where $\iota_{i}: \bar{M}_{i} \rightarrow \bar{W}$ are the inclusions.

One can now pass from Steenrod to Čech homology. There is a natural transformation

$$
{ }^{s} \mathrm{H}_{d}(X) \rightarrow \check{\mathrm{H}}_{d}(X)
$$

defined on the category $\mathcal{A}_{L C}$ (cf. Appendix $\mathrm{A}$ ). While Cech homology does not satisfy the exactness axiom, it has a property which does not hold for singular homology that we will make crucial use of:

- Čech Continuity Property: For every inverse system of compact Hausdorff spaces with inverse limit $X$, the maps $X \rightarrow X_{\alpha}$ induce a natural isomorphism

$$
\check{\mathrm{H}}_{*}(X) \stackrel{\cong}{\longleftrightarrow} \lim _{\longleftarrow} \check{\mathrm{H}}_{*}\left(X_{\alpha}\right)
$$

(cf. [ES, Thm. X.3.1]). Applying (1.8) to the classes in (1.6) yields Čech theory fundamental classes, which we will usually write as simply

$$
[M] \in \check{\mathrm{H}}_{d}(M ; G), \quad[\bar{M}] \in \check{\mathrm{H}}_{d}(\bar{M} ; G) .
$$

Equations (1.6) and (1.7) continue to hold in Cech theory. (If desired, one could further push these classes into the dual of compactly supported Čech cohomology; see 4.4 and Remark 5.0.2 of [Pd].) 
Appendix B shows that one can replace Steenrod by Borel-Moore homology in (1.4) (Lemma B.1 with $X=S$ ). It also shows that condition (1.4) holds if the Lebesgue covering dimension of $S$ satisfies

$$
\operatorname{dim} S \leq d-2,
$$

and this is true if $S$ is covered by manifolds of dimension $\leq d-2$ in the sense of Lemma B.3. This provides a practical method to verify (1.4). For example, if $M$ is a smooth complex quasiprojective variety with positive dimension, then its closure $\bar{M}$ and its 1-point compactification $M^{+}$are both thin compactifications.

1.2. Families. These ideas extend naturally to families. Consider a proper continuous map

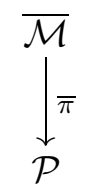

which we regard as a family of spaces, namely the fibers $\overline{\mathcal{M}}_{p}=\bar{\pi}^{-1}(p)$ for $p \in \mathcal{P}$. Let $\mathcal{K}_{\mathcal{P}}$ denote the category whose objects are continuous maps $\varphi: K \rightarrow \mathcal{P}$ where $K$ is a nonempty, compact, path-connected Hausdorff space, and whose morphisms are commutative diagrams

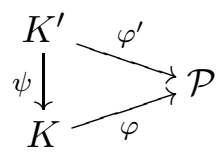

of continuous maps. Each map $\varphi: K \rightarrow \mathcal{P}$ in $\mathcal{K}_{\mathcal{P}}$ gives a pullback diagram (1.15a) of proper continuous maps, where

$$
\overline{\mathcal{M}}_{\varphi}=\{(k, x) \in K \times \overline{\mathcal{M}} \mid \varphi(k)=\bar{\pi}(x)\}
$$

is the fiber product of $\varphi$ and $\bar{\pi}$. Similarly, the morphism (1.13) gives the diagram (1.15b).
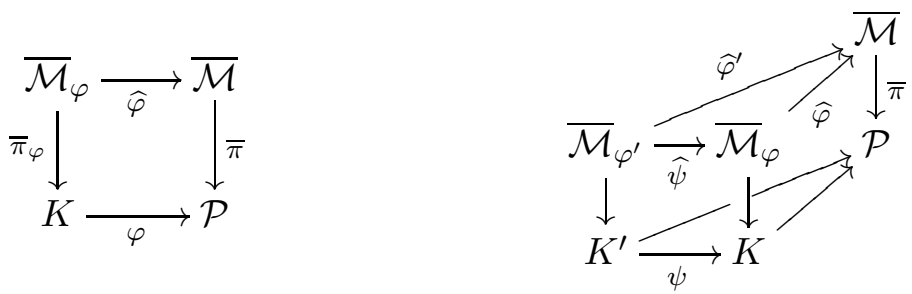

1.3. Relative homology functors. For each $d \in \mathbb{Z}$, Čech homology determines a functor

$$
\check{\mathrm{H}}_{d}: \mathcal{K}_{\mathcal{P}} \rightarrow \mathcal{A} b
$$

with values in the category $\mathcal{A} b$ of abelian groups by $\varphi \mapsto \check{\mathrm{H}}_{d}\left(\overline{\mathcal{M}}_{\varphi}\right)$.

Definition 1.2. A lift of (1.16) to a functor

$$
\mu: \mathcal{K}_{\mathcal{P}} \rightarrow \mathcal{A} b^{*}
$$

into the category of abelian groups with a distinguished element is called a relative homology functor of degree $d$ associated to $\bar{\pi}: \overline{\mathcal{M}} \rightarrow \mathcal{P}$. 
Thus a relative homology functor assigns to each continuous map $\varphi: K \rightarrow \mathcal{P}$ from a non-empty, compact, path-connected Hausdorff space $K$ an element

$$
\mu(\varphi) \in \check{\mathrm{H}}_{d}\left(\overline{\mathcal{M}}_{\varphi}\right)
$$

such that each morphism (1.13) induces an equality

$$
\widehat{\psi}_{*}\left[\mu\left(\varphi^{\prime}\right)\right]=\mu(\varphi) .
$$

Two special cases are especially important:

(i) For each $p \in \mathcal{P}$, we can take $\varphi$ to be the inclusion of $p$ into $\mathcal{P}$. Then $\overline{\mathcal{M}}_{\varphi}$ is identified with the fiber $\overline{\mathcal{M}}_{p}$ over $p$, and we obtain a class

$$
\mu(p) \in \check{\mathrm{H}}_{d}\left(\overline{\mathcal{M}}_{p}\right) .
$$

(ii) For each path $\gamma:[0,1] \rightarrow \mathcal{P}$ with endpoints $p$ and $q$, let $\iota_{0}\left(\right.$ resp. $\left.\iota_{1}\right)$ be the inclusion $p \mapsto 0 \in[0,1]$ (resp. $q \mapsto 1 \in[0,1]$ ). Applying (1.18) first with $\psi=\iota_{0}$ and $\varphi=\gamma$, then with $\psi=\iota_{1}$ and $\varphi=\gamma$, yields the consistency condition

$$
\left(\iota_{0}\right)_{*} \mu(p)=\left(\iota_{1}\right)_{*} \mu(q) \text { in } \check{\mathrm{H}}_{d}\left(\overline{\mathcal{M}}_{\gamma}\right) .
$$

The importance of these two cases is reflected in the following extension and uniqueness result. The proof is an application of the Cech continuity property (1.9).

Proposition 1.3. Let $\bar{\pi}: \overline{\mathcal{M}} \rightarrow \mathcal{P}$ be a proper continuous map from a Hausdorff space to a locally path-connected metrizable space.

(a) (Uniqueness) If two relative homology functors associated to $\bar{\pi}$ are equal on a dense set of points $p \in \mathcal{P}$, then they are equal.

(b) (Extension) Suppose that there is a dense subset $\mathcal{P}^{*}$ of $\mathcal{P}$, and an assignment

$$
p \mapsto \mu(p) \in \check{\mathrm{H}}_{d}\left(\overline{\mathcal{M}}_{p}\right)
$$

defined for $p \in \mathcal{P}^{*}$ such that, for any $p, q \in \mathcal{P}^{*}$, (1.19) holds for each path $\gamma:[0,1] \rightarrow \mathcal{P}$ in a $C^{0}$ dense subset of the space of paths in $\mathcal{P}$ from $p$ to $q \in \mathcal{P}^{*}$. Then (1.20) extends uniquely to a relative homology functor.

Proof. (a) Relative homology functors satisfy (1.18), and hence (1.19). Statement (a) therefore follows from the uniqueness in statement (b).

(b) After fixing a metric on $\mathcal{P}$, the hypotheses of (b) are the same as those of Extension Lemma 3.4 of [IP1, which shows that (1.20) extends uniquely to an assignment, still denoted $p \mapsto \mu(p)$, defined for all $p \in \mathcal{P}$ and satisfying (1.19) for all paths $\gamma$ with endpoints $p, q \in \mathcal{P}$. Then, given a map $\varphi: K \rightarrow \mathcal{P}$ in $\mathcal{K}_{\mathcal{P}}$, choose a point $p \in K$ and set

$$
\mu(\varphi)=\widehat{\iota}_{*} \mu(p) \in \check{\mathrm{H}}_{d}\left(\overline{\mathcal{M}}_{\varphi}\right),
$$

where $\widehat{\iota}$ is the inclusion $\overline{\mathcal{M}}_{p} \hookrightarrow \overline{\mathcal{M}}_{\varphi}$. Given another point $q \in K$, choose a path $\sigma:[0,1] \rightarrow K$ from $p$ to $q$. Applying (1.19) to the path $\gamma=\varphi \circ \sigma$ and pushing forward in homology by the map $\widehat{\sigma}: \overline{\mathcal{M}}_{\gamma} \rightarrow \overline{\mathcal{M}}_{\varphi}$ shows that the class (1.21) is independent of the choice of $p$. With this established, (1.18) follows from (1.21) by applying $\widehat{\psi}_{*}$.

Finally, this extension is unique: two relative homology functors $\mu$ and $\mu^{\prime}$ that agree on all points $p \in \mathcal{P}^{*}$ must also agree for all $p \in \mathcal{P}$ by the uniqueness of [IP1, Lemma 3.4]. But then they agree for all $\varphi$ in $\mathcal{K}_{\mathcal{P}}$ : applying (1.18), for both $\mu$ and $\mu^{\prime}$, to the inclusion of any point $p \hookrightarrow K$ shows that $\mu(\varphi)=\widehat{\iota}_{*} \mu(p)=\widehat{\iota}_{*} \mu^{\prime}(p)=\mu^{\prime}(\varphi)$. 
We next note four functorial constructions. All four start with a relative homology functor $\mu$ of degree $d$ on a family $\bar{\pi}: \overline{\mathcal{M}} \rightarrow \mathcal{P}$ as in (1.12).

1.4. Pullbacks. For each continuous map $\sigma: \mathcal{Q} \rightarrow \mathcal{P}$ from a space $\mathcal{Q}$, there is an associated pullback family and a commutative square

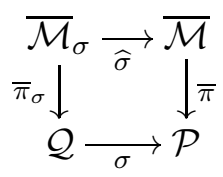

where, one can check, $\bar{\pi}_{\sigma}$ is proper. Then there is an induced pullback relative homology functor $\sigma^{*} \mu$ : for each $\varphi: K \rightarrow \mathcal{Q}$ in $\mathcal{K}_{\mathcal{Q}}$, the composition $\sigma \circ \varphi$ is in $\mathcal{K}_{\mathcal{P}}$, and we define

$$
\left(\sigma^{*} \mu\right)(\varphi)=\mu(\sigma \circ \varphi)
$$

in the Čech homology, using the identification of $\left(\overline{\mathcal{M}}_{\sigma}\right)_{\varphi}$ with $\overline{\mathcal{M}}_{\sigma \circ \varphi}$, defined by the projection $\left(\overline{\mathcal{M}}_{\sigma}\right)_{\varphi} \rightarrow \overline{\mathcal{M}}_{\sigma \circ \varphi}$. Thus defined, $\sigma^{*} \mu$ satisfies (1.18), so is a relative homology functor of degree $d$ associated to $\bar{\pi}_{\sigma}$.

\subsection{Pushforwards and Invariants. Given a continuous map}

$$
\bar{f}: \overline{\mathcal{M}} \rightarrow Z
$$

to a Hausdorff space $Z$, one obtains numerical invariants as follows.

Corollary 1.4. For $p \in \mathcal{P}$ and $\beta \in \check{\mathrm{H}}^{*}(Z ; \mathbb{Z})$, the class

$$
\bar{f}_{*} \mu(p) \in \check{\mathrm{H}}_{d}(Z ; \mathbb{Z})
$$

and the function

$$
I_{\beta}(p)=\left\langle\bar{f}_{*} \mu(p), \beta\right\rangle
$$

are independent of $p$ on path-connected components of $\mathcal{P}$.

Proof. Any pair $p, q$ of points in the same path-connected component of $\mathcal{P}$ are the endpoints of a path $\gamma:[0,1] \rightarrow \mathcal{P}$. By (1.19), $\bar{f}_{*} \mu(p)$ is equal to $\bar{f}_{*} \mu(q)$ in the image of $\bar{f}_{*} \gamma_{*}: \check{\mathrm{H}}_{d}\left(\overline{\mathcal{M}}_{\gamma}\right) \rightarrow$ $\check{\mathrm{H}}_{d}(Z)$, and hence $I_{\beta}(p)=I_{\beta}(q)$.

1.6. Cap Products. Suppose that (1.12) is such that there is a cap product

$$
\cap: \check{\mathrm{H}}_{d}(X) \otimes \check{\mathrm{H}}^{k}(X) \rightarrow \check{\mathrm{H}}_{d-k}(X)
$$

defined on a category of spaces $X$ that includes all $\overline{\mathcal{M}}_{\varphi}$ for $\varphi \in \mathcal{K}_{\mathcal{P}}$, and which has the naturality property $f_{*} a \cap \xi=f_{*}\left(a \cap f^{*} \xi\right)$ for all maps $f: X \rightarrow Y$ in that category. Then, given a relative homology functor $\mu$, each cohomology element $\alpha \in \check{\mathrm{H}}^{*}(\overline{\mathcal{M}})$ determines a relative homology functor $\mu \cap \alpha$ defined by

$$
(\mu \cap \alpha)(\varphi)=\mu(\varphi) \cap \widehat{\varphi}^{*} \alpha,
$$

for each $\varphi \in \mathcal{K}_{\mathcal{P}}$. Indeed, for maps $\varphi^{\prime}=\varphi \circ \psi$ and $\widehat{\varphi}^{\prime}=\widehat{\varphi} \circ \widehat{\psi}$ as in (1.13) and (1.15)(b), the naturality of $\cap$ and formula (1.18) for $\mu$ imply that

$$
\widehat{\psi}_{*}\left[(\mu \cap \alpha)\left(\varphi^{\prime}\right)\right]=\widehat{\psi}_{*}\left[\mu\left(\varphi^{\prime}\right) \cap \widehat{\psi}^{*}\left(\widehat{\varphi}^{*} \alpha\right)\right]=\left[\widehat{\psi}_{*} \mu\left(\varphi^{\prime}\right)\right] \cap\left(\widehat{\varphi}^{*} \alpha\right)=(\mu \cap \alpha)(\varphi) .
$$

Thus $\mu \cap \alpha$ satisfies (1.18), so is a relative homology functor.

Sections 5 and 6 describe how the invariants (1.23) and the cap products (1.24) are related to intersection numbers. 
1.7. Relative fundamental classes. Following Definition 3.1 of [IP1], we now impose additional structure on the map $\bar{\pi}: \overline{\mathcal{M}} \rightarrow \mathcal{P}$. In particular, we assume that $\mathcal{P}$ is locally path-connected and metrizable, and is a Baire space.

Definition 1.5. We say that a family $\bar{\pi}: \overline{\mathcal{M}} \rightarrow \mathcal{P}$ as in (1.12) is a relatively thin family if $\mathcal{P}$ is a locally path-connected metrizable Baire space, and there is a number $d$ and a second category set $\mathcal{P}^{*} \subseteq \mathcal{P}$ such that for each $p, q \in \mathcal{P}^{*}$

(a) $\overline{\mathcal{M}}_{p}$ is a thin compactification of the d-dimensional oriented manifold $\mathcal{M}_{p}=\pi^{-1}(p)$.

(b) The space of continuous paths in $\mathcal{P}$ from $p$ to $q$ contains a $C^{0}$-dense subset of paths $\gamma$ for which the fiber product $\overline{\mathcal{M}}_{\gamma}$ is a thin cobordism from $\overline{\mathcal{M}}_{p}$ to $\overline{\mathcal{M}}_{q}$.

These conditions ensure that the generic fiber $\overline{\mathcal{M}}_{p}$ of $\bar{\pi}$ has a fundamental class as in (1.10). This leads to the notion of a relative fundamental class, which is our key object of study.

Definition 1.6. A relative fundamental class (RFC) of a relatively thin family is a relative homology functor $\mu$ of degree $d$ such that

$$
\mu(p)=\left[\overline{\mathcal{M}}_{p}\right]
$$

for each $p$ in the set $\mathcal{P}^{*}$. We will often write $\mu(\varphi)$ as $\left[\overline{\mathcal{M}}_{\varphi}\right]^{r f c}$.

This is equivalent to Definition 4.1 of [P1]: one direction is clear, the other follows immediately from Proposition 1.3(b).

Note that a RFC is not a single element of the homology of some space. Rather, it is a functor that, as in (1.17), assigns to each continuous map $\varphi: K \rightarrow \mathcal{P}$ from a non-empty, compact path-connected space $K$, a Čech homology class

$$
\mu(\varphi)=\left[\overline{\mathcal{M}}_{\varphi}\right]^{r f c} \in \check{\mathrm{H}}_{d}\left(\overline{\mathcal{M}}_{\varphi}\right)
$$

that satisfies a naturality axiom and a normalization axiom:

A1. Every triangle (1.13) of continuous maps, where $K$ and $L$ are nonempty, compact, and path-connected, induces an equality

$$
\widehat{\psi}_{*}\left[\overline{\mathcal{M}}_{\varphi^{\prime}}\right]^{r f c}=\left[\overline{\mathcal{M}}_{\varphi}\right]^{r f c} .
$$

A2. For each $p \in \mathcal{P}^{*}$,

$$
\left[\overline{\mathcal{M}}_{p}\right]^{r f c} \text { is the fundamental class }\left[\overline{\mathcal{M}}_{p}\right] .
$$

Finally, we note that relative fundamental classes are natural under certain changes of the parameter space $\mathcal{P}$ (cf. [IP1, Section 6]). A morphism between relatively thin families $\bar{\pi}$ and $\bar{\pi}^{\prime}$ is a diagram of continuous maps

$$
\begin{gathered}
\overline{\mathcal{M}} \underset{\widehat{f}}{\longrightarrow} \overline{\mathcal{N}} \\
\bar{\pi} \downarrow \\
\stackrel{\mathcal{P}}{\underset{f}{\longrightarrow}} \stackrel{\downarrow \mathcal{Q} .}{ } .
\end{gathered}
$$

We say that a morphism (1.28) is generically degree 1 if there exist second category subsets $\mathcal{P}^{*}$ of $\mathcal{P}$ and $\mathcal{Q}^{*}$ of $\mathcal{Q}$ satisfying the condition of Definition 1.5(a), with $f\left(\mathcal{P}^{*}\right) \subseteq \mathcal{Q}^{*}$, and such for each $p \in \mathcal{P}^{*}, \widehat{f}$ restricts to a degree 1 map

$$
\widehat{f}_{p}: \mathcal{M}_{p} \rightarrow \mathcal{N}_{f}(p) .
$$


Lemma 1.7. If a morphism (1.28) of relatively thin families is generically degree 1, then

$$
\mu^{r f c}=f^{*} \nu^{r f c} \text {. }
$$

Proof. If $p \in \mathcal{P}^{*}$ and $q=f(p) \in \mathcal{Q}^{*}$, then

$$
\mu^{r f c}(p)=\left[\overline{\mathcal{M}}_{p}\right] \quad \text { and } \quad\left(f^{*} \nu^{r f c}\right)(p)=\nu^{r f c}(q)=\left[\overline{\mathcal{N}}_{q}\right] .
$$

But $\left(\widehat{f}_{p}\right)_{*}\left[\overline{\mathcal{M}}_{p}\right]=\left[\overline{\mathcal{N}}_{q}\right]$ by assumption (1.29). Thus $f^{*} \nu^{r f c}(p)=\mu^{r f c}(p)$ for a dense set of points $p$ in $\mathcal{P}$. The lemma then follows by Proposition 1.3(a).

The pullback property (1.30) is not true for general morphisms (1.28); the two relative homology functors may not even have the same dimension. In particular, if one restricts the space $\mathcal{P}$ of parameters to a submanifold of $\mathcal{P}$, the relative fundamental classes need not correspond. Examples of this phenomenon are given in Section 6 of [IP1].

The constructions in this section required two homology theories. We first used the $e x$ actness of Steenrod homology to extend fundamental classes from manifolds to their thin compactifications; we then passed to Čech homology and used its continuity property to extend fundamental classes to all fibers in a family. Unfortunately, on the category of compact pairs with $\mathbb{Z}$ coefficients, no homology theory is both exact and continuous [ES, §X.4].

On the other hand, with coefficients in $\mathbb{Q}$, Steenrod and Čech homology are naturally isomorphic on the category $\mathcal{A}_{C}$ of compact spaces and continuous maps, giving a single theory that is both exact and continuous (and essentially unique - see $\S$ A.2 of the appendix). The constructions of this section then produce an RFC in rational Steenrod homology. This approach avoids Čech homology, at the expense of losing track of whether the invariants (1.23) are integers.

\section{Fredholm Families and Relative fundamental Classes}

We now define and focus attention on a class of families (1.12) where the structures of Section 1 arise naturally via the Sard-Smale theorem. The motivating examples occur in gauge theories, where one has universal moduli spaces $\mathcal{M}$ which are Banach manifolds and have compactifications $\overline{\mathcal{M}}$ with natural maps to a manifold $\mathcal{P}$ of parameters. The definitions and results of this section codify the relevant structure of such moduli spaces which is needed to ensure the existence of a relative fundamental class.

In this and later sections, the term "Banach manifold" means a metrizable separable Banach manifold, finite or infinite dimensional. Such manifolds are second countable and paracompact (metrizability is needed to apply the dimension theory in Appendix B). By a Fredholm family we mean a Fredholm map

$$
\begin{gathered}
\mathcal{M} \\
\downarrow_{\mathcal{P}} \\
\mathcal{P}
\end{gathered}
$$

between $C^{l}$ Banach manifolds, finite or infinite dimensional, which we again regard as a family of spaces (the fibers of $\pi$ ) parameterized by $\mathcal{P}$. Such a map $\pi$ has an associated Fredholm index $d$, and we assume that

$$
l>\max (d+1,0) .
$$

We also assume that (2.1) comes equipped with two additional structures: 
- A relative orientation specified by a nowhere zero section of the relative determinant bundle. A relative orientation on $\mathcal{M}$ induces an orientation on each regular fiber $\mathcal{M}_{p}$.

- A (metrizable) relative compactification, meaning a metrizable space $\overline{\mathcal{M}}$ together with a commutative diagram

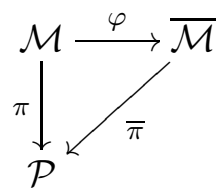

where $\varphi$ is an inclusion of $\mathcal{M}$ as an open subset, and $\bar{\pi}: \overline{\mathcal{M}} \rightarrow \mathcal{P}$ is continuous and proper.

We call the set $\mathcal{S}=\overline{\mathcal{M}} \backslash \mathcal{M}$ the singular locus of $\overline{\mathcal{M}}$, so $\overline{\mathcal{M}}$ is the disjoint union

$$
\overline{\mathcal{M}}=\mathcal{M} \cup \mathcal{S} .
$$

The following definition generalizes and supersedes the notion of a "Fredholm-stratified thin compactification" defined in [IP1, and casts it in terms of three easily-verifiable conditions. (Definition 5.2 in [IP1 is a special case in which each $\varphi_{\alpha}$ is an inclusion and the images $\varphi_{\alpha}\left(\mathcal{S}_{\alpha}\right)$ are disjoint.)

Definition 2.1. Fix a relatively oriented Fredholm family (2.1) of index d. A Fredholm thin compactification of $\mathcal{M}$ is a metrizable relative compactification as in (2.3), together with a countable set $\mathcal{A}$ and, for each $\alpha \in \mathcal{A}$, a diagram

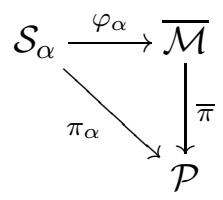

such that

(1) $\pi_{\alpha}: \mathcal{S}_{\alpha} \rightarrow \mathcal{P}$ is a Fredholm family of index $d_{\alpha} \leq d-2$.

(2) Each $\varphi_{\alpha}$ is either (i) continuous and locally injective, or (ii) locally Lipschitz.

(3) $\left\{\varphi_{\alpha}\left(\mathcal{S}_{\alpha}\right) \mid \alpha \in \mathcal{A}\right\}$ cover $\mathcal{S}$.

These conditions imply that the fibers $\overline{\mathcal{M}}_{p}$ and the cobordisms $\overline{\mathcal{M}}_{\gamma}$ are compact metrizable spaces.

In this context, the Sard-Smale theorem yields a crucial fact:

Lemma 2.2. A Fredholm thin compactification (2.4) is a relatively thin family.

Proof. Because $\mathcal{P}$ is a separable metrizable Banach manifold, it is locally path-connected, and is a Baire space. It remains to verify conditions (a) and (b) of Definition 1.5.

(a) The Sard-Smale theorem, applied to $\pi$ and to each $\pi_{\alpha}, \alpha \in \mathcal{A}$, shows that there are second category subsets $\mathcal{P}_{0}$ and $\mathcal{P}_{\alpha}$ of $\mathcal{P}$ such that (i) the fiber $\mathcal{M}_{p}$ over each $p \in \mathcal{P}_{0}$ is a $C^{1}$ manifold of dimension $d$ (with orientation induced from the relative orientation), and (ii) the fiber $\mathcal{S}_{\alpha, p}$ of $\pi_{\alpha}$ over each $p \in \mathcal{P}_{\alpha}$ is a $C^{1}$ manifold of dimension $d_{\alpha} \leq d-2$. Then

$$
\mathcal{P}^{*}=\mathcal{P}_{0} \cap \bigcap \mathcal{P}_{\alpha}
$$

is also a second category subset of $\mathcal{P}$. We call elements of $\mathcal{P}^{*}$ the regular values of $\bar{\pi}$. 
For each $p \in \mathcal{P}^{*}, \mathcal{S}_{p}=\overline{\mathcal{M}}_{p} \backslash \mathcal{M}_{p}$ is a closed, hence compact, subset of $\overline{\mathcal{M}}_{p}$. By Definition 2.1, $\mathcal{S}_{p}$ is covered by the sets $\varphi_{\alpha}\left(\mathcal{S}_{\alpha, p}\right)$. Each $\mathcal{S}_{\alpha, p}$ is a finite-dimensional submanifold of the (second countable metrizable) Banach manifold $\mathcal{S}_{\alpha}$, so is $\sigma$-compact. Assumption (2) and Lemma B.3 then show that

$$
{ }^{s} \mathrm{H}_{k}\left(\mathcal{S}_{p}\right)=0 \quad \forall k>d-2 .
$$

Thus $\overline{\mathcal{M}}_{p}$ is a metrizable thin compactification of $\mathcal{M}_{p}$.

(b) Now fix $p, q \in \mathcal{P}^{*}$ and $l$ satisfying (2.2). Because any continuous path is the $C^{0}$ limit of $C^{l}$ paths, it suffices to show that (b) holds for a dense subset of the space $\mathcal{P}^{l}(p, q)$ of $C^{l}$ paths $\gamma:[0,1] \rightarrow \mathcal{P}$ from $p$ to $q$. Furthermore, noting that $p$ and $q$ are regular values of $\pi$, the Sard-Smale theorem shows that the set of $\gamma \in \mathcal{P}^{l}(p, q)$ that are transverse to $\pi$ is open and dense, and the same is true with $\pi$ replaced by $\pi_{\alpha}$ for each $\alpha$. The intersection of these sets is a dense subset of paths $\gamma \in \mathcal{P}^{l}(p, q)$ for which (i) the fiber product (1.14) is a $(d+1)$-dimensional oriented manifold

$$
\mathcal{M}_{\gamma}
$$

whose boundary is canonically identified with $\mathcal{M}_{p} \sqcup \mathcal{M}_{q}$, and (ii) each fiber product $\left(\mathcal{S}_{\alpha}\right)_{\gamma}$ is a manifold of dimension $d_{\alpha}+1 \leq d-1$ with boundary $\mathcal{S}_{\alpha, \partial \gamma}=\mathcal{S}_{\alpha, p} \sqcup \mathcal{S}_{\alpha, q}$ and with maps

$$
\text { id } \times \varphi_{\alpha}:\left(\mathcal{S}_{\alpha}\right)_{\gamma} \rightarrow \overline{\mathcal{M}}_{\gamma}
$$

also satisfying property (2) of Definition 2.1, By Definition 2.1](3) and the commutativity of diagram (2.4), the images of the maps (2.6) cover the singular locus of $\overline{\mathcal{M}}_{\gamma}$ and the images of their restrictions to $\mathcal{S}_{\alpha, \partial \gamma}$ cover the singular locus of the boundary $\overline{\mathcal{M}}_{\partial \gamma}=\overline{\mathcal{M}}_{p} \sqcup \overline{\mathcal{M}}_{q}$. We can then similarly apply Lemma B.3 to conclude that the singular locus of $\overline{\mathcal{M}}_{\gamma}$ satisfies correct properties to be a thinly compactified cobordism from $\overline{\mathcal{M}}_{p}$ to $\overline{\mathcal{M}}_{q}$.

Combining Lemma 2.2 and Proposition 1.3 leads to our first main theorem.

Theorem 2.3. A Fredholm thin compactification $\bar{\pi}: \overline{\mathcal{M}} \rightarrow \mathcal{P}$ admits a unique relative fundamental class.

Proof. First apply Lemma 2.2, noting that the set $\mathcal{P}^{*}$ in (2.5) of regular values of $\bar{\pi}$ is dense in $\mathcal{P}$ by the Baire Category theorem. Then, as noted after Definition 1.5, $\overline{\mathcal{M}}_{p}$ has a Cech fundamental class $\left[\overline{\mathcal{M}}_{p}\right]$ for each $p \in \mathcal{P}^{*}$. Define an assignment (1.20) by setting

$$
\mu(p)=\left[\overline{\mathcal{M}}_{p}\right] \in \check{\mathrm{H}}_{d}\left(\overline{\mathcal{M}}_{p}\right)
$$

for $p \in \mathcal{P}^{*}$. For any $q \in \mathcal{P}^{*}$, Lemma 2.2 also shows that there is a dense set of paths $\gamma$ from $p$ to $q$, each with an associated thin cobordism $\overline{\mathcal{M}}_{\gamma}$. Applying (1.7) to this cobordism shows that the consistency condition (1.19) for these paths $\gamma$.

Proposition 1.3 now applies ( $\mathcal{P}$ is a separable Banach manifold, so is locally connected and metrizable). Thus the assignment $p \mapsto \mu(p)$ extends uniquely to a relative homology functor that satisfies (2.7).

Remark 2.4. Suppose that a family as in Theorem 2.3 contains a complex algebraic subfamily, meaning that there is a diagram (1.22) where $\sigma: \mathcal{Q} \rightarrow \mathcal{P}$ is an inclusion and $\bar{\pi}_{\sigma}$ is a proper complex algebraic map between varieties. As in $\S 1.4$, the RFC on $\overline{\mathcal{M}}$ pulls back to a relative homology functor on $\overline{\mathcal{M}}_{\sigma}$, so for each proper algebraic map $\varphi: K \rightarrow \mathcal{Q}$, one obtains a Čech homology class

$$
\left[\overline{\mathcal{M}}_{\sigma \circ \varphi}\right]^{r f c} \text {. }
$$


Because $\overline{\mathcal{M}}_{\sigma \circ \varphi}$ is compact and locally contractible, its Steenrod, Čech, and Borel-Moore homologies are isomorphic with $\mathbb{Z}$ coefficients (cf. $\S$ A.3). Thus (2.8) can be regarded as a class in Borel-Moore homology, which is more commonly used by algebraic geometers.

Note that the class (2.8) is defined even if $\overline{\mathcal{M}}_{\sigma \circ \varphi}$ has no regular fibers, and it has the naturality property (1.26) with respect to proper algebraic base changes with path-connected base. However, its existence and uniqueness depend on the existence of a non-algebraic object: the Fredholm thin compactification of Theorem 2.3.

\section{Proper maps ANd PSEUdo-Cycles}

In geometric topology, intersection invariants can be defined using pseudo-cycles. This section gives a general definition of pseudo-cycle, relates it to the definition used in [MS], and describes how pseudo-cycle classes can be realized by pushing forward the fundamental class of a thinly compactified manifold. As in [IP1] and [Sw], we work in Steenrod homology with coefficients in $\mathbb{Z}$ or $\mathbb{Q}$, although the results and proofs hold equally well in Borel-Moore homology (see Appendix $\mathrm{A}$ and Lemma B.1). The connection with intersection numbers is made in the next section.

3.1. Proper maps. In Steenrod and Borel-Moore homology, fundamental classes push forward only under proper continuous maps. Thus it is helpful to describe two ways (Lemmas 3.1 and 3.2 ) that a continuous map

$$
f: M \rightarrow Z
$$

between Hausdorff spaces can be modified to produce a proper map. Recall that the Omega limit set of $f$ is defined to be

$$
\Omega_{f}=\bigcap_{K \subseteq M} \overline{f(M \backslash K)}
$$

where the bar denotes closure and the intersection is over all compact sets $K \subseteq M$.

Lemma 3.1. For any subset $A$ of $Z \backslash \Omega_{f}$, the restriction of $f$ to $M_{A}=f^{-1}(A)$ is a proper map

$$
f_{A}: M_{A} \rightarrow A
$$

Proof. Fix a compact set $C \subseteq A$. We must show that the closed set $f_{A}^{-1}(C)$ is compact. This is true if $f_{A}^{-1}(C)$ lies in some compact $K \subseteq M$. Otherwise, for each compact $K \subseteq M$, the set $B_{K}=C \cap \overline{f(M \backslash K)}$ is a non-empty, closed - hence compact - subset of $C$. But then $\bigcap_{K} B_{K}=C \cap \Omega_{f}$ is non-empty, contradicting the fact that $C \cap \Omega_{f} \subseteq A \cap \Omega_{f}=\emptyset$.

Instead of restricting $f$, we can extend it.

Lemma 3.2. Suppose that $M$ is a subset of a compact space $\bar{M}$ with closed complement $S=\bar{M} \backslash M$. If $\bar{f}: \bar{M} \rightarrow Z$ is a continuous extension of (3.1), then $\bar{f}$ is proper and $\Omega_{f} \subseteq \bar{f}(S)$.

Proof. The first conclusion is evident because a continuous map from a compact space to a Hausdorff space is proper. For the second, suppose by contradiction that there is a point $y \in \Omega_{f}$ that is not in $\bar{f}(S)$. The hypothesis implies that $S$ is compact and hence so is $\bar{f}(S)$. Hence we can find disjoint open neighborhoods $U$ of $y$ and $V$ of $\bar{f}(S)$. Then $K=\bar{M} \backslash \bar{f}^{-1}(V)$ is a compact subset of $M$, and $f(M \backslash K) \subseteq \bar{f}(\bar{M} \backslash K) \subseteq V$. But this implies that $y \notin \overline{f(M \backslash K)}$, so $y \notin \Omega_{f}$, giving a contradiction. 
3.2. Pseudo-cycles. A $d$-dimensional pseudo-cycle is a continuous map

$$
f: M \rightarrow Z
$$

from an oriented $d$-dimensional topological manifold $M$ to a locally compact metric space $Z$ such that $f(M)$ has compact closure and

$$
\operatorname{dim} \Omega_{f} \leq d-2
$$

where dim denotes the Lebesgue covering dimension. These conditions imply that $\Omega_{f}$ is compact and hence, by Lemma B.1.

$$
{ }^{s} \mathrm{H}_{k}\left(\Omega_{f} ; \mathbb{Z}\right)=0 \quad \text { for all } k \geq d-1 .
$$

This notion of pseudo-cycle generalizes the one used in [MS], cf. Lemma 3.5] below. Note that if $f$ is proper, then $\Omega_{f}=\emptyset$, hence $f$ is a pseudo-cycle.

Two such pseudo-cycles $f_{i}: M_{i} \rightarrow Z, i=1,2$, are called cobordant if there exists a $(d+1)$ dimensional oriented manifold $W$ with boundary $\partial W=M_{2} \sqcup\left(-M_{1}\right)$ and a map $F: W \rightarrow Y$ such that

$$
\left.F\right|_{M_{1}}=f_{1},\left.\quad F\right|_{M_{2}}=f_{2}, \quad \operatorname{dim} \Omega_{F} \leq d-1, \text { and } \bar{F}(W) \text { is compact. }
$$

The following result is due to M. Schwarz [Sw, Theorem 3.1].

Lemma 3.3. A pseudo-cycle (3.3) determines a Steenrod class

$$
[f] \in{ }^{s} \mathrm{H}_{d}(X ; \mathbb{Z}),
$$

where $X=\overline{f(M)} \subseteq Z$. It is defined by formula (3.10) below.

Proof. By Lemma 3.1, $f$ restricts to a proper map $f^{o}: M^{o} \rightarrow X \backslash \Omega_{f}$ whose domain

$$
M^{o}=f^{-1}\left(Z \backslash \Omega_{f}\right)
$$

is an open subset of the oriented $d$-manifold $M$. Hence $M^{o}$ has a fundamental class which satisfies

$$
\left[M^{o}\right]=\rho_{o}[M]
$$

(see (1.3)), where $\rho_{o}=\rho_{M^{o}}$.

The long exact sequence (1.2), together with (3.5), shows that the restriction to $U=Z \backslash \Omega_{f}$ induces an isomorphism

$$
0 \longrightarrow{ }^{s} \mathrm{H}_{d}(X) \stackrel{\rho}{\cong}{ }^{s} \mathrm{H}_{d}\left(X \backslash \Omega_{f}\right) \longrightarrow 0 .
$$

Thus the image of $[M]$ under the composition

$$
{ }^{s} \mathrm{H}_{d}(M) \stackrel{\rho_{o}}{\longrightarrow}{ }^{s} \mathrm{H}_{d}\left(M^{o}\right) \stackrel{f_{*}^{o}}{\longrightarrow}{ }^{s} \mathrm{H}_{d}\left(X \backslash \Omega_{f}\right) \stackrel{\rho^{-1}}{\cong}{ }^{s} \mathrm{H}_{d}(X) .
$$

determines a class

$$
[f]=\rho^{-1} f_{*}^{o}\left[M^{o}\right]=\rho^{-1} f_{*}^{o} \rho_{o}[M] \in{ }^{s} \mathrm{H}_{d}(X ; \mathbb{Z}) .
$$


If $f$ is a proper map then its pseudo-cycle class (3.7) is simply

$$
[f]=f_{*}[M] .
$$

More generally, if $f$ is a pseudo-cycle and $U$ is an open subset of $Z \backslash \Omega_{f}, f_{U}: M_{U} \rightarrow U$ is proper by Lemma 3.1, and

$$
\rho_{U}[f]=\left[f_{U}\right] \quad \text { in }{ }^{s} \mathrm{H}_{d}(U) .
$$

This follows from the commutative diagram obtained by restricting (3.9) over $U$ :

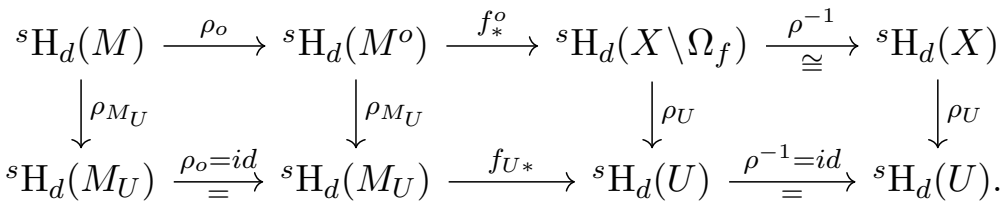

Lemma 3.4. If $F: W \rightarrow Z$ is a cobordism between d-dimensional pseudo-cycles $f_{1}$ and $f_{2}$, then

$$
\left[f_{1}\right]=\left[f_{2}\right] \quad \text { in }{ }^{s} \mathrm{H}_{d}(Y),
$$

where $Y=\overline{F(W)}$ is the closure of the image of the cobordism.

Proof. As in (3.5), the assumptions (3.6) imply that ${ }^{s} \mathrm{H}_{d}\left(\Omega_{F}\right)=0$. Then (1.2) shows that the restriction to the open subset $Y^{o}=Y \backslash \Omega_{F}$ of $Y$ is an injection:

$$
0={ }^{s} \mathrm{H}_{d}\left(\Omega_{F}\right) \longrightarrow{ }^{s} \mathrm{H}_{d}(Y) \stackrel{\rho_{Y o}}{\longrightarrow}{ }^{s} \mathrm{H}_{d}\left(Y \backslash \Omega_{F}\right) .
$$

Therefore it suffices to prove the equality of the restrictions of (3.13) to $Y^{o}$.

By Lemma 3.1, the restriction $F^{o}: W^{o} \rightarrow Y^{o}$ of $F$ over $Y^{o}$ is proper. But

$$
W^{o}=F^{-1}\left(Y^{o}\right)=W \backslash F^{-1}\left(\Omega_{F}\right)
$$

is an open subset of $W$, so is a manifold with boundary $\partial W^{o}=\partial W \cap W^{o}=\partial W \backslash F^{-1}\left(\Omega_{F}\right)$. Moreover, as in the proof of [IP1, Lemma 2.10], under the long exact sequence sequence

$$
{ }^{s} \mathrm{H}_{d+1}\left(W^{o}\right) \stackrel{\rho}{\longrightarrow}{ }^{s} \mathrm{H}_{d+1}\left(W^{o} \backslash \partial W^{o}\right) \stackrel{\partial}{\longrightarrow}{ }^{s} \mathrm{H}_{d}\left(\partial W^{o}\right) \stackrel{\iota *}{\longrightarrow}{ }^{s} \mathrm{H}_{d}\left(W^{o}\right)
$$

we have $\partial\left[W^{o} \backslash \partial W^{o}\right]=\left[\partial W^{o}\right]$, and hence $\iota_{*}\left[\partial W^{o}\right]=0$. Pushing forward by the proper map $F^{o}: W^{o} \rightarrow Y^{o}$ gives

$$
F_{*}^{o}\left[\partial W^{o}\right]=0 \quad \text { in }{ }^{s} \mathrm{H}_{d}\left(Y^{o}\right) .
$$

Because $\partial W=M_{2} \sqcup\left(-M_{1}\right)$, we have

$$
\partial W^{o}=\partial W \backslash F^{-1}\left(\Omega_{F}\right)=M_{2}^{o} \sqcup\left(-M_{1}^{o}\right),
$$

where each $M_{i}^{\circ}=f_{i}^{-1}\left(Y^{o}\right)=M_{i} \backslash f_{i}^{-1}\left(\Omega_{F}\right)$ is an open subset of $M_{i}$ and therefore a submanifold. But the restriction $f_{i}^{o}$ of $f_{i}$ over $Y^{o}$ is equal to the restriction of $F^{o}$ to $M_{i}^{o}$, and therefore

$$
F_{*}^{o}\left[\partial W^{o}\right]=F_{*}^{o}\left[M_{2}^{o}\right]-F_{*}^{o}\left[M_{1}^{o}\right]=\left[f_{2}^{o}\right]-\left[f_{1}^{o}\right]=\rho_{Y^{o}}\left(\left[f_{2}\right]-\left[f_{1}\right]\right) .
$$

Combining this with (3.14) shows that (3.13) holds after restriction to $Y^{o}$, completing the proof. 
3.3. A pseudo-cycle criterion. In practice, one needs a method for verifying condition (3.4) for continuous maps

$$
f: M \rightarrow Z
$$

with $M$ and $Z$ as in (3.3). To that end, we impose various regularity conditions on $\Omega_{f}$.

Pseudo-cycle criterion. Assume that $\Omega_{f}$ is covered by the images of countably many maps $\varphi_{n}: U_{n} \rightarrow Z$ where, for each $n$, each $U_{n}$ is a $\sigma$-compact topological manifold of dimension $\leq d-2$, and at least one of the following holds:

(a) $\varphi_{n}$ is continuous and locally injective,

(b) $\varphi_{n}$ is locally Lipschitz,

(c) $\varphi_{n}$ is a $C^{1}$ map between $C^{1}$ manifolds.

Lemma B.3 immediately implies:

Lemma 3.5. Any map (3.15) that satisfies the pseudo-cycle criterion is a pseudo-cycle.

Note that no higher regularity on $f$ itself is needed. Sometimes Condition (c), with $C^{1}$ replaced by $C^{\infty}$, is used in the definition of pseudo-cycle in place of (3.4) (cf. [MS, §6.5]).

3.4. Pseudo-cycles and thin compactifications. Pseudo-cycles and maps from thin compactifications both determine Steenrod homology classes. The next lemma gives conditions under which these classes coincide; the corresponding fact for families is given in Theorem 5.2 .

Lemma 3.6. If a map $f: M \rightarrow Z$ as in (3.3) extends to a continuous map $\bar{f}: \bar{M} \rightarrow Z$ from a thin compactification $\bar{M}=M \cup S$ and $\operatorname{dim} \bar{f}(S) \leq d-2$, then $f$ is a pseudo-cycle and

$$
[f]=\bar{f}_{*}[\bar{M}] \text { in }{ }^{s} \mathrm{H}_{d}(X),
$$

where $X=\bar{f}(\bar{M})$ is the image of $\bar{f}$.

Proof. Because $S$ is compact (cf. Definition [1.1), the assumptions imply that $\bar{f}(S)$ is a compact subset of the metric space $Z$, while $\Omega_{f} \subseteq \bar{f}(S)$ by Lemma 3.2. Lemma B.2 a) then shows that $\operatorname{dim} \Omega_{f} \leq \operatorname{dim} \bar{f}(S) \leq d-2$, so $f$ is a pseudo-cycle.

As in (3.5) and (3.8), the assumption that $\operatorname{dim} \bar{f}(S) \leq d-2$ implies that the restriction to $U=X \backslash \bar{f}(S)$

$$
{ }^{s} \mathrm{H}_{d}(X) \stackrel{\rho_{U}}{\cong}{ }^{s} \mathrm{H}_{d}(U)
$$

is an isomorphism. Thus it suffices to show that the two sides of (3.16) are equal when restricted to $U$. Using the notation of (3.2), the restriction of $\bar{f}$ over $U$ is equal to the restriction $f_{U}: M_{U} \rightarrow U$ of $f$ over $U$ (because $\left.\bar{f}^{-1}(U) \subseteq \bar{M} \backslash S=M\right)$. Therefore

$$
\rho_{U} \bar{f}_{*}[\bar{M}]=f_{U *} \rho_{M_{U}}[\bar{M}]=f_{U *}\left[M_{U}\right],
$$

which is equal to $\rho_{U}[f]$ by (3.11). 


\section{Intersection PAIRINGs of Pseudo-CyCles}

In an oriented differentiable manifold, there are two ways to define the intersection between two pseudo-cycles $f$ and $g$ of complementary dimension. A geometric intersection $f \cdot g$ is obtained by perturbing the maps to make them (strongly) transverse, and then counting the intersection points with sign (cf. [MS, $\S 6.5]$ ). A homological intersection $[f] \bullet[g]$ is obtained by applying the intersection pairing in Steenrod homology to the pseudo-cycles classes defined by Lemma 3.3. After reviewing these definitions, we give conditions under which these two intersection pairings are equal.

Throughout this section, all manifolds are $C^{1}$, oriented, finite-dimensional and $\sigma$-compact (as in [MS, §6.5]). Each such manifold is separable and metrizable, and admits a proper embedding into euclidean space (see Appendix $\mathrm{A}$ ). We fix one such manifold $N$ of dimension $n$, and consider $C^{1}$ maps

$$
f: M \rightarrow N \quad g: P \rightarrow N
$$

from manifolds $M$ and $P$. We will call these " $C^{1}$ pseudo-cycles" if they are pseudo-cycles in the sense of McDuff and Salamon [MS, §6.5]. Thus a $C^{1}$ map $f: M \rightarrow N$ between oriented manifolds is a $C^{1}$ pseudo-cycle if

(i) $\overline{f(M)}$ is compact, and

(ii) $\Omega_{f}$ is covered by the image of a $C^{1}$-map $f^{\prime}: M^{\prime} \rightarrow N$ with $\operatorname{dim} M^{\prime} \leq \operatorname{dim} M-2$.

4.1. Geometric intersections. As in [MS, §6.5], we say that two $C^{1}$ pseudo-cycles $f$ and $g$ are strongly transverse if $f$ is transverse to $g$ as $C^{1}$-maps, and

$$
\Omega_{f} \cap \overline{g(P)}=\emptyset, \quad \Omega_{g} \cap \overline{f(M)}=\emptyset .
$$

If, in addition, $f$ and $g$ have complementary dimensions $(\operatorname{dim} M+\operatorname{dim} P=\operatorname{dim} N)$, then

$$
Z=\{(x, y) \mid f(x)=g(y)\}=(f \times g)^{-1}(\Delta)
$$

is a compact oriented 0 -dimensional manifold. The geometric intersection number is then defined by

$$
f \cdot g=\sum_{z \in Z} \sigma(z)
$$

where $\sigma(z)= \pm 1$ is the sign of the local intersection of $f$ and $g$ at $f(x)=g(y)$. In fact, they show that given two complementary dimension $C^{1}$-pseudo-cycles, then $g$ can be perturbed (by a $C^{1}$-small diffeomorphism of the target) to make it strongly transverse to $f$, and that the resulting intersection number

$$
f \cdot g \in \mathbb{Z}
$$

is independent of choices, and depends only on the cobordism classes of $f$ and $g$ [MS, Lemma 6.5.5].

4.2. Homological intersections. Appendix A relates Steenrod to other homology theories and describes features of Steenrod homology beyond those described in Section 1. Using coefficients $\mathbb{Z}$ or $\mathbb{Q}$, for any closed subset $X$ of the manifold $N$ as in (4.1), there are natural isomorphisms

$$
{ }^{s} \mathrm{H}_{*}(X) \cong H^{n-*}(N, N \backslash X) \cong H_{*}^{B M}(X)
$$

between Steenrod homology, relative singular cohomology, and Borel-Moore homology (A.3) and (A.4). By these isomorphisms, the intersection theory facts stated in Appendix $\mathrm{A}$ for 
Borel-Moore homology carry over to Steenrod homology. Thus, while we work with Steenrod homology, all results in this section hold with Steenrod replaced by Borel-Moore homology.

Under (4.5), the cup product in singular cohomology corresponds to a Borel-Moore intersection pairing (A.28), and hence to a Steenrod intersection pairing

$$
{ }^{s} \mathrm{H}_{d}(X) \otimes{ }^{s} \mathrm{H}_{k}(Y) \stackrel{\bullet}{\longrightarrow}{ }^{s} \mathrm{H}_{d+k-n}(X \cap Y) .
$$

This is natural under restriction as in (A.30): for each open subset $U$ of $N$

$$
\rho_{U}(a \bullet b)=\rho_{U}(a) \bullet \rho_{U}(b) .
$$

In particular, if $X \cap Y$ is compact and we use $\mathbb{Z}$ coefficients, we get an intersection number

$$
a \cdot b=\varepsilon(a \bullet b) \in \mathbb{Z} .
$$

between classes of complementary dimensions (cf. (‥34) $)$.

4.3. Relating intersection pairings. Now consider two $C^{1}$ pseudo-cycles (4.1) with complementary dimensions $d$ and $n-d$. By Lemma 3.3, these determine homology classes

$$
[f] \in{ }^{s} \mathrm{H}_{d}(X), \quad[g] \in{ }^{s} \mathrm{H}_{n-d}(Y),
$$

in the closed subsets $X=\overline{f(M)}$ and $Y=\overline{g(P)}$ of $N$. The $C^{1}$ pseudo-cycle condition (i) above ensures that $X \cap Y$ is compact, so there is a homological intersection number

$$
[f] \cdot[g] \in \mathbb{Z},
$$

defined by (4.8), which we can compare to the geometric intersection number (4.4).

Proposition 4.1. If $f$ and $g$ as in (4.1) are $C^{1}$ pseudo-cycles of complementary dimension, then their geometric and homological intersection numbers are equal:

$$
f \cdot g=[f] \cdot[g] .
$$

Proof. First assume that $f$ and $g$ are strongly transverse. Then by (4.2), we can choose an open neighborhood $U$ of $X \cap Y$ which is disjoint from $\Omega_{f} \cup \Omega_{g}$. Restricting to $U$ and using (4.7), we have

$$
[f] \bullet[g]=\rho_{U}([f] \bullet[g])=\rho_{U}[f] \bullet \rho_{U}[g] .
$$

Because $X \cap U$ is an open subset of $X$ that does not intersect $\Omega_{f}$, Lemma 3.1 and equation (3.11) imply that $f$ restricts to a proper map $f_{U}: f^{-1}(U) \rightarrow U$ with

$$
\rho_{U}[f]=\left[f_{U}\right],
$$

and similarly for $g$. A direct computation (see Example A.4) shows that, with the notation of (4.3),

$$
\left[f_{U}\right] \cdot\left[g_{U}\right]=\sum_{z \in Z} \sigma(z)=f \cdot g .
$$

Thus (4.9) holds in the case that $f$ and $g$ are strongly transverse.

For the general case, note that both sides of (4.9) are invariant under proper cobordism. Indeed, if $G: W \rightarrow N$ is a cobordism between $g$ and $g^{\prime}$ whose image has compact closure $B=\overline{G(W)}$, then

$$
[g]=\left[g^{\prime}\right] \quad \text { in }{ }^{s} \mathrm{H}_{k}(B)
$$

by Lemma 3.4. Therefore

$$
[f] \bullet[g]=[f] \bullet\left[g^{\prime}\right] \quad \text { in }{ }^{s} \mathrm{H}_{0}(B \cap X),
$$


where $X=\overline{f(M)}$ and where $B \cap X$ is compact. We conclude that $[f] \cdot[g]$, like (4.4), is invariant under proper cobordisms of $g$.

Finally, as in Lemma 6.5.5 of [MS], one can perturb $g$ (by a compactly supported diffeomorphism of the target that is isotopic to the identity) to make $g$ strongly transverse to $f$. This gives a cobordism $G$, and the proposition follows.

4.4. Pairing with singular homology. Returning to the manifold $N$ in (4.1), there is also a natural transformation

$$
\varphi: H_{*}(N ; \mathbb{Z}) \rightarrow{ }^{s} \mathrm{H}_{*}(N ; \mathbb{Z})
$$

from singular to Steenrod homology (cf. (A.5)). Every class in the image of $\varphi$ can be represented by a pseudo-cycle. The construction, given in [Mas, $\S 9.6$ and $\S 10.2$ ] and [MS, Remark 6.5.3], can be summarized as follows.

Each $b \in H_{k}(N ; \mathbb{Z})$ can be represented by a singular cycle $\bar{g}: Q \rightarrow N$ from a $k$-dimensional finite simplicial complex without boundary. After smoothing across the $(k-1)$-faces, we can assume that $Q=M \cup S$, where $M$ is an oriented $k$-dimensional $C^{1}$ manifold and $S$ is the $(k-2)$-skeleton. Then (4.10) is defined by

$$
\varphi(b)=\bar{g}_{*}[Q] .
$$

By further smoothing, we can assume that the restriction of $\bar{g}$ to each simplex of $Q$ is $C^{1}$, and therefore the restriction of $\bar{g}$ to $M$ is a $C^{1}$-pseudo-cycle

$$
g=\left.\bar{g}\right|_{M}: M \rightarrow N
$$

In this setting, McDuff and Salamon associate to each $C^{1}$ pseudo-cycle $f: M \rightarrow N$ a homomorphism

$$
\Phi_{f}: H_{*}(N ; \mathbb{Z}) \rightarrow \mathbb{Z}
$$

from singular homology defined by the geometric intersection pairing (4.3):

$$
\Phi_{f}(b)=f \cdot g .
$$

They show that this is independent of the representative $\bar{g}$ of $b$ and its smoothing [MS, Lemma 6.5.6]. In fact, $\Phi_{f}$ can be written terms of the topological intersection pairing (4.8) in Steenrod homology as follows.

Proposition 4.2. For each $C^{1}$ pseudo-cycle $f$, the pairing (4.14) satisfies

$$
\Phi_{f}(b)=[f] \cdot \varphi(b) \quad \forall b \in H_{*}(N ; \mathbb{Z}) .
$$

Proof. Given $b \in H_{k}(N ; \mathbb{Z})$, choose a map $\bar{g}: Q \rightarrow N$ as in (4.11) and a smoothing as in (4.12). Note that $Q$ is the disjoint union of $M$ and the $(d-2)$-skeleton $S$ of $Q$, so $Q$ is a thin compactification of $M$. Moreover, $g(S)$ is covered by the images of $C^{1}$-maps from the cells of the $(d-2)$-dimensional skeleton of $Q$. Hence, by Lemmas 3.5 and 3.6, the pseudo-cycle class of $g$ is

$$
[g]=\bar{g}_{*}[Q]=\varphi(b) .
$$

Then by (4.14) and Proposition 4.1, $\Phi_{f}(b)=f \cdot g=[f] \cdot[g]=[f] \cdot \varphi(b)$.

Finally, if $N$ is closed, (4.15) can be translated into a pairing in singular theory: 
Corollary 4.3. Let $N$ be a closed manifold as in (4.1). Then for each $C^{1}$ pseudo-cycle $f$, there is a singular homology class $a_{f}$ such that $\varphi\left(a_{f}\right)=[f]$ and

$$
\Phi_{f}(b)=a_{f} \cdot b=\left\langle b, P D^{-1} a_{f}\right\rangle
$$

for each $b \in H_{*}(N ; \mathbb{Z})$, where $P D: H^{*}(N) \rightarrow H_{*}(N)$ is Poincaré duality.

Proof. For compact manifolds, (4.10) is an isomorphism (cf. (A.8)), so $[f]=\varphi\left(a_{f}\right)$ for some unique $a_{f} \in H_{*}(N)$. Starting with this class $a_{f}$, we can repeat the procedure of (4.11) and (4.12) to obtain an $\bar{h}: P \rightarrow N$, such that, as in (4.16),

$$
[h]=\bar{h}_{*}[P]=\varphi\left(a_{f}\right)=[f],
$$

where the $C^{1}$-pseudo-cycle $h$ is the restriction of $\bar{h}$ to the complement of the codimension 2 skeleton of $P$. Then

$$
\Phi_{f}(b)=[f] \cdot \varphi(b)=\Phi_{h}(b)=h \cdot g
$$

by (4.14) and (4.15). But after a further perturbation, $\bar{h}$ and $\bar{g}$ are transversely intersecting singular cycles that represent $a_{f}$ and $b$. Hence

$$
h \cdot g=\bar{h}_{*}[P] \cdot g_{*}[Q]=a_{f} \cdot b=\left\langle b, P D^{-1} a_{f}\right\rangle,
$$

using the fact that, in singular theory, intersection numbers depend only on homology and are given by Poincaré duality (we use the same sign conventions as in (A.35).

\section{INTERSECTIONS AND THIN FAMILIES}

We now apply the results of Sections 3 and 4 to Fredholm thin families, and show that the invariants defined in Corollary 1.4 using relative fundamental classes are equal to those defined in terms of intersections of pseudo-cycles, as is done, for example, in [MS]. All of the spaces in this section and the next are assumed to be metrizable, and we assume that $N$ is a compact manifold without boundary, so there are natural isomorphisms (see (A.8))

$$
H_{*}(N ; \mathbb{Z}) \stackrel{s}{\longrightarrow} \mathrm{H}_{*}(N ; \mathbb{Z}) \stackrel{\gamma}{\cong} \check{\mathrm{H}}_{*}(N ; \mathbb{Z}) .
$$

Fix a relatively oriented Fredholm family of index $d$ (cf. (2.1)), together with a $C^{1}$ map $f$ to a closed oriented $C^{1}$ manifold $N$ of dimension $n$ :

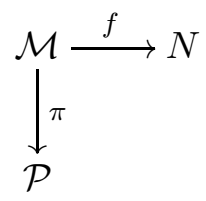

Also fix a Fredholm thin compactification $\overline{\mathcal{M}}$ of $\mathcal{M}$ as in Definition 2.1 ,

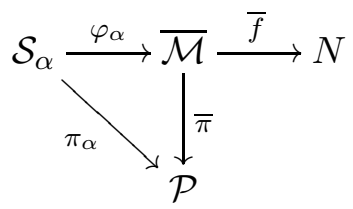

and let $\mathcal{P}^{*} \subset \mathcal{P}$ be the set of regular parameters defined in (2.5). In this section we consider continuous maps $\bar{f}: \overline{\mathcal{M}} \rightarrow N$ as in the above diagram, and use the following terminology. 
Definition 5.1. For a map $\bar{f}: \overline{\mathcal{M}} \rightarrow N$ as in (5.2), we say $\bar{f}$ is $C^{m}$ if the composition $\bar{f} \circ \varphi_{\alpha}: \mathcal{S}_{\alpha} \rightarrow N$ is $C^{m}$ for each $\alpha$ (including $\mathcal{S}_{0}=\mathcal{M}$ ).

In this situation, Theorem 2.3 implies that each fiber $\overline{\mathcal{M}}_{p}$ of $\bar{\pi}$ has a relative fundamental class $\left[\overline{\mathcal{M}}_{p}\right]^{r f c} \in \check{\mathrm{H}}_{d}\left(\overline{\mathcal{M}}_{p}\right)$. As in (1.23), this class pushes forward and pairs with Čech cohomology, defining a homomorphism

$$
I_{p}: \check{\mathrm{H}}^{*}(N ; \mathbb{Z}) \rightarrow \mathbb{Z}
$$

by

$$
I_{p}(\beta)=\left\langle\bar{f}_{*}\left[\overline{\mathcal{M}}_{p}\right]^{r f c}, \beta\right\rangle=\left\langle\left[\overline{\mathcal{M}}_{p}\right]^{r f c}, \bar{f}^{*} \beta\right\rangle .
$$

By Corollary 1.4, $I_{p}$ is independent of $p$ on each path-connected component of $\mathcal{P}$.

The number (5.3) is often written as

$$
\int_{\left[\overline{\mathcal{M}}_{p}\right]^{r f c}} \bar{f}^{*} \beta .
$$

It is commonly interpreted either

(1) in terms of intersections with pseudo-cycles in $N$ that represent homology classes Poincaré dual to $\beta$, or

(2) in terms of "cutdown" spaces.

The next theorem shows how the relative fundamental class approach is compatible with interpretation (1). Section 6 addresses compatibility with interpretation (2).

Given maps as in diagram (5.2) and $p \in \mathcal{P}^{*}$, let $\bar{f}_{p}: \overline{\mathcal{M}}_{p} \rightarrow N$ be the restriction of $\bar{f}$ to the fiber of $\bar{\pi}$ over $p$, and its further restriction $f_{p}: \mathcal{M}_{p} \rightarrow N$.

Theorem 5.2. Suppose that the map $\bar{f}$ in (5.2) is $C^{1}$ in the sense of Definition 5.1. Then for each $p \in \mathcal{P}^{*}$,

(a) $f_{p}$ is a $C^{1}$ pseudo-cycle and its pseudo-cycle class (3.7) satisfies

$$
\gamma\left[f_{p}\right]=\bar{f}_{*}\left[\overline{\mathcal{M}}_{p}\right]^{r f c}
$$

in $\check{\mathrm{H}}_{d}(N ; \mathbb{Z})(c f .(5.1))$.

(b) If $N$ is compact, the topological invariant (5.3) is related to the geometric intersection pairing (4.13) by

$$
I_{p}(\beta)=(-1)^{d(n-d)} \Phi_{f_{p}}(b),
$$

where $b=D \beta \in H_{n-d}(N)$ is the Poincaré dual of $\beta$ under (A.23).

Proof. (a) Fix a regular value $p \in \mathcal{P}^{*}$. By Lemma $2.2, \overline{\mathcal{M}}_{p}$ is a thin compactification of the oriented $d$-dimensional manifold $\mathcal{M}_{p}$ with singular locus $\mathcal{S}_{p}=\overline{\mathcal{M}}_{p} \backslash \mathcal{M}_{p}$. It therefore has a Steenrod fundamental class $\left[\overline{\mathcal{M}}_{p}\right]$ and a corresponding Cech class $\gamma\left[\overline{\mathcal{M}}_{p}\right]$. The naturality of $\gamma$ and the normalization axiom (1.27) then imply that

$$
\bar{f}_{*}\left[\overline{\mathcal{M}}_{p}\right]^{r f c}=\gamma\left(\left(\bar{f}_{p}\right)_{*}\left[\overline{\mathcal{M}}_{p}\right]\right) \quad \text { in } \check{\mathrm{H}}_{d}(N ; \mathbb{Z}) .
$$

For each $\alpha$, as in the proof of Lemma 2.2, the fiber $\pi_{\alpha}^{-1}(p)=\left(\mathcal{S}_{\alpha}\right)_{p}$ is a $\sigma$-compact $C^{1}$ manifold of dimension at most $d-2$. Furthermore, the restriction of $\bar{f} \circ \varphi_{\alpha}$ to $\left(\mathcal{S}_{\alpha}\right)_{p}$ is $C^{1}$ and

$$
\bar{f}_{p}\left(\mathcal{S}_{p}\right) \subseteq \bigcup_{\alpha}\left(\bar{f}_{p} \circ \varphi_{\alpha}\right)\left(\mathcal{S}_{\alpha}\right)_{p}
$$


Lemma B.3 then shows that the compact set $\bar{f}_{p}\left(\mathcal{S}_{p}\right)$ has dimension at most $d-2$. Therefore, by Lemma 3.6, $f_{p}: \mathcal{M}_{p} \rightarrow N$ is a $C^{1}$ pseudo-cycle, and

$$
\left[f_{p}\right]=\left(\bar{f}_{p}\right)_{*}\left[\overline{\mathcal{M}}_{p}\right]
$$

in ${ }^{s} \mathrm{H}_{d}(N)$. Together with (5.6), this yields (5.4).

(b) Returning to (5.3) and using (5.4), and then using (A.14) to switch from the Cech to the Steenrod Kronecker pairing, we have

$$
I_{p}(\beta)=\left\langle\gamma\left[f_{p}\right], \beta\right\rangle=\left\langle\left[f_{p}\right], \beta\right\rangle .
$$

This last expression can be written as an intersection pairing as in (A.35). Hence, using the definition of $b$ and (4.15), we have

$$
I_{p}(\beta)=D \beta \cdot\left[f_{p}\right]=(-1)^{d(n-d)}\left[f_{p}\right] \cdot b=(-1)^{d(n-d)} \Phi_{f}(b) .
$$

\section{RFCs for Cutdown Families}

We now turn to the second interpretation of the intersection number (5.3). The basic idea is that for a generic submanifold $V$ of $N$, the inverse image of the map $\bar{f}: \overline{\mathcal{M}} \rightarrow N$ should be a "cutdown" family $\overline{\mathscr{V}} \rightarrow \mathcal{P}$ that has all of the properties of the original relatively thin family $\overline{\mathcal{M}} \rightarrow \mathcal{P}$; in particular it should have a relative fundamental class. We will show that this is true provided $f$ is "fully transverse to $V$ ". If, in addition, the cutdown family has index 0 , the invariant (5.3) has the expected geometric interpretation: it is the signed number of elements of a generic fiber of this index 0 family, i.e. those in a generic fiber of the original family whose images under $f$ lie in $V$.

This section gives the details. We begin with a standard formula (6.4) that relates the fundamental classes of a manifold and a submanifold. We then extend it first to (metrizable) thin compactifications of finite-dimensional manifolds, and then to Fredholm thin families.

6.1. Orientation classes. Let $X$ be a closed subset of a locally compact space $Z$. Then there is a cap product

$$
H_{d}^{B M}(Z) \otimes H^{k}(Z, Z \backslash X) \stackrel{\cap}{\longrightarrow} H_{d-k}^{B M}(X)
$$

in Borel-Moore homology with coefficients in $\mathbb{Z}$ or $\mathbb{Q}$ (see (A.17) ) with the naturality properties (A.18)-(A.20).

Now suppose that $N$ is an oriented $C^{1}$ manifold of dimension $n$ and

$$
V \hookrightarrow N
$$

is a properly embedded oriented submanifold of codimension $k$. The orientations determine Borel-Moore fundamental classes $[N] \in H_{n}^{B M}(N)$ and $[V]$ respectively (see (A.21)), and the Thom class of the normal bundle defines an orientation class

$$
u=u_{V, N} \in H^{k}(N, N \backslash V)
$$

in singular cohomology (see (A.24) $)$. As in (A.25), these are related by

$$
[V]=[N] \cap u_{V, N} \text { in } H_{n-k}^{B M}(V) .
$$


6.2. Thin compactifications. Formula (6.4) extends to metrizable thin compactifications as follows. Fix $V \subseteq N$ and $u$ as above. Suppose that $\bar{V} \subseteq \bar{N}$ are thin compactifications of $V$ and $N$ such that

(i) $V=\bar{V} \cap N$, and

(ii) the orientation class (6.3) is the restriction to $N$ of some class $\bar{u} \in H^{*}(\bar{N}, \bar{N} \backslash \bar{V})$, i.e. $u=j_{N}^{*} \bar{u}$ where $j_{N}: N \rightarrow \bar{N}$ is the inclusion.

Lemma 6.1. If (i) and (ii) above hold and $\bar{N}$ is metrizable, then

$$
[\bar{V}]=[\bar{N}] \cap \bar{u} \quad \text { in } H_{*}^{B M}(\bar{V} ; \mathbb{Z})={ }^{s} \mathrm{H}_{*}(\bar{V} ; \mathbb{Z}) .
$$

Proof. Because $\bar{N}$ and $\bar{V}$ are compact and metrizable, their Steenrod and Borel-Moore homologies are isomorphic [BM, $\S 5]$ (see also $\S$ A.3 below). For the proof, we work in Borel-Moore homology, using (6.4) and noting that equations (1.1)-(1.6) hold in Borel-Moore homology (cf. $§ \mathrm{~A} .2$ and Lemma B.1). In particular, the restriction $\rho_{V}: H_{n-k}^{B M}(\bar{V}) \rightarrow H_{n-k}^{B M}(V)$ is an isomorphism, $\rho_{V}[\bar{V}]=[V]$ and $\rho_{N}[\bar{N}]=[N]$. Therefore it suffices to show that the two sides of (6.5) are equal after applying $\rho_{V}$. But assumption (i), and the naturality (A.20) of the cap product with respect to the restriction to $N$, and assumption (ii) imply that

$$
\rho_{V}([\bar{N}] \cap \bar{u})=\rho_{N \cap \bar{V}}([\bar{N}] \cap \bar{u})=\rho_{N}[\bar{N}] \cap j_{N}^{*}(\bar{u})=[N] \cap u
$$

in $H_{*}^{B M}(V)$, and this is equal to $[V]=\rho_{V}[\bar{V}]$ by (6.4).

6.3. Cutdown families. Fix a map $\bar{f}: \overline{\mathcal{M}} \rightarrow N$ as in (5.2) and a codimension $k$ submanifold $V$ of $N$ as in (6.2). This data determines a "cutdown" family

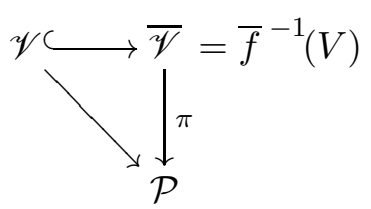

obtained by restricting $\bar{\pi}$ to $\bar{f}^{-1}(V)$, setting $\mathscr{V}=\overline{\mathscr{V}} \cap \mathcal{M}$, and giving $\mathscr{V}$ the induced relative orientation. Recall that the index $d$ family $\pi: \mathcal{M} \rightarrow \mathcal{P}$ satisfies (2.2), and we assume that $f$ is $C^{m}$, where

$$
m>\max (d-k, 0) .
$$

Definition 6.2. We say that $\bar{f}: \overline{\mathcal{M}} \rightarrow N$ is fully transverse to a submanifold $V$ if $\bar{f} \circ \varphi_{\alpha}$ is transverse to $V$ for all $\alpha$.

Lemma 6.3. With $N, V$ as above, if a map $\bar{f}$ as in (5.2) is $C^{m}$ and fully transverse to $V$, then the cutdown family (6.6) is a Fredholm thin compactification of index $d-k$.

Proof. By assumption, each composition $f_{\alpha}=\bar{f} \circ \varphi_{\alpha}$ in (5.2) is a $C^{m}$ map transverse to $V$. Therefore $\mathscr{V}_{\alpha}=f_{\alpha}^{-1}(V)$ is a closed codimension $k$ submanifold of $\mathcal{S}_{\alpha}$, and $\bar{\pi}$ restricts to a Fredholm map $\mathscr{V}_{\alpha} \rightarrow \mathcal{P}$ whose index is (index $\pi_{\alpha}$ ) $-k$. In particular, the top stratum $\mathscr{V}=\overline{\mathscr{V}} \cap \mathcal{M} \rightarrow \mathcal{P}$ is a Fredholm family of index $d-k$, and all other $\mathscr{V}_{\alpha}$ have index at most $d-k-2$. This implies that the restriction of $\bar{\pi}$ to $\overline{\mathscr{V}}$ is a Fredholm thin compactification of $\mathscr{V}$ with index $d-k$ (cf. Definition 2.1). 
6.4. The cutdown RFC. In the remainder of this section, we will extend formula (6.5) to cutdown families. For this, we pass to rational coefficients and use the natural identifications

$$
{ }^{s} \mathrm{H}_{*}(X ; \mathbb{Q})=H_{*}^{B M}(X ; \mathbb{Q})=\check{\mathrm{H}}_{*}(X ; \mathbb{Q})
$$

for compact metric spaces (see A.7). Under this identification (6.1) becomes a cap product in rational Čech homology.

For each parameter $p \in \mathcal{P}$, let $\overline{\mathscr{V}}_{p}$ and $\overline{\mathcal{M}}_{p}$ denote the fibers of the two families (6.6) and (5.2) respectively, and $\bar{f}_{p}: \overline{\mathcal{M}}_{p} \rightarrow N$ the restriction of $\bar{f}$ to $\overline{\mathcal{M}}_{p}$. Then the pullback of (6.3) is a class

$$
\bar{f}_{p}^{*} u \in H^{k}\left(\overline{\mathcal{M}}_{p}, \overline{\mathcal{M}}_{p} \backslash \overline{\mathscr{V}}_{p}\right),
$$

and hence the cap product (6.1) induces a map

$$
\cap \bar{f}_{p}^{*} u: \check{\mathrm{H}}_{d}\left(\overline{\mathcal{M}}_{p} ; \mathbb{Q}\right) \longrightarrow \check{\mathrm{H}}_{d-k}\left(\overline{\mathscr{V}}_{p} ; \mathbb{Q}\right) \text {. }
$$

Proposition 6.4. Let $V \hookrightarrow N$ as in (6.2) with orientation class (6.3). Assume a map $\bar{f}$ as in (5.2) is $C^{1}$ and fully transverse to $V$. Then the cutdown family (6.6) has a relative fundamental class

$$
\left[\overline{\mathscr{V}_{p}}\right]^{r f c} \in \check{\mathrm{H}}_{*}\left(\overline{\mathscr{V}_{p}} ; \mathbb{Z}\right)
$$

which is related to the relative fundamental class of $\overline{\mathcal{M}}$ by

$$
\left[\overline{\mathscr{V}}_{p}\right]^{r f c}=\left[\overline{\mathcal{M}}_{p}\right]^{r f c} \cap \bar{f}_{p}^{*} u
$$

in $\check{\mathrm{H}}_{*}\left(\overline{\mathscr{V}_{p}} ; \mathbb{Q}\right)=H_{*}^{B M}\left(\overline{\mathscr{V}_{p}} ; \mathbb{Q}\right)$ for all $p \in \mathcal{P}$.

Proof. Lemma 6.3 and Theorem 2.3 immediately imply the existence of the relative fundamental class (6.9). To establish (6.10), apply Lemma 2.2 to both $\overline{\mathcal{M}}$ and $\overline{\mathscr{V}}$ and intersect the resulting sets (2.5) of regular values. This yields a second category subset $\mathcal{P}^{* *}$ of $\mathcal{P}$ such that, for each $p \in \mathcal{P}^{* *}$,

(i) $\overline{\mathcal{M}}_{p}$ is a metrizable thin compactification of $\mathcal{M}_{p}$.

(ii) $\overline{\mathscr{V}}_{p}$ is a metrizable thin compactification of $\mathscr{V}_{p}$.

(iii) $\mathscr{V}_{p}=f_{p}^{-1}(V)$ is a oriented embedded submanifold of $\mathcal{M}_{p}$ whose orientation class, as in (A.26), is $f_{p}^{*} u$, where $u=u_{N, V}$.

Now fix $p \in \mathcal{P}^{* *}$. The naturality of the restriction map shows that $f_{p}^{*} u$ is the restriction to $\mathcal{M}_{p}$ of the class (6.7). Therefore Lemma 6.1 applies, giving

$$
\left[\overline{\mathscr{V}}_{p}\right]=\left[\overline{\mathcal{M}}_{p}\right] \cap \bar{f}_{p}^{*} u \quad \text { in } H_{*}^{B M}\left(\overline{\mathscr{V}}_{p}, \mathbb{Q}\right)=\check{\mathrm{H}}_{*}\left(\overline{\mathscr{V}}_{p}, \mathbb{Q}\right),
$$

for each $p \in \mathcal{P}^{* *}$. To complete the proof, observe that each side of (6.11) is a relative homology functor associated to $\overline{\mathscr{V}} \rightarrow \mathcal{P}$ :

- By Theorem 2.3. $\mu=[\overline{\mathscr{V}}]^{r f c}$ is a relative homology functor with

$$
\mu(p)=\left[\overline{\mathscr{V}}_{p}\right]^{r f c} \in \check{\mathrm{H}}_{*}\left(\overline{\mathscr{V}}_{p}, \mathbb{Q}\right)
$$

- The calculation (1.25) used to show that (1.24) is relative homology functor, now using the cap product (6.8) with its naturality property (A.18), shows that $\tilde{\mu}=[\overline{\mathcal{M}}]^{r f c} \cap \bar{f}^{*} u$ is a relative homology functor with

$$
\tilde{\mu}(p)=\left[\overline{\mathcal{M}}_{p}\right]^{r f c} \cap \bar{f}_{p}^{*} u \in \check{\mathrm{H}}_{*}\left(\overline{\mathscr{V}}_{p}, \mathbb{Q}\right)
$$


But (6.11) shows that

$$
\mu(p)=\tilde{\mu}(p) \quad \text { for all } p \in \mathcal{P}^{* *} .
$$

By Proposition 1.3(a), we conclude that $\mu$ and $\tilde{\mu}$ are equal, giving (6.10).

Corollary 6.5. Under the assumptions of Proposition 6.4

$$
\int_{\left[\overline{\mathscr{Y}}_{p}\right]^{r f c}} \iota^{*} \alpha=\int_{\left[\overline{\mathcal{M}}_{p}\right]^{r f c}} \bar{f}_{p}^{*} u \cup \alpha \quad \forall \alpha \in H^{*}\left(\overline{\mathcal{M}}_{p} ; \mathbb{Q}\right)
$$

where $\iota$ is the inclusion $\overline{\mathscr{V}}_{p} \hookrightarrow \overline{\mathcal{M}}_{p}$.

Proof. Applying the naturality formula (A.19) and (6.10) gives

$$
\left[\overline{\mathscr{V}}_{p}\right]^{r f c} \cap \iota^{*} \alpha=\left(\left[\overline{\mathcal{M}}_{p}\right]^{r f c} \cap \bar{f}_{p}^{*} u\right) \cap \iota^{*} \alpha=\left[\overline{\mathcal{M}}_{p}\right]^{r f c} \cap\left(\bar{f}_{p}^{*} u \cup \alpha\right),
$$

which is equivalent to (6.12) (cf. (A.35) ).

\section{VFCS DEFINED BY IMPLICIT ATLASES}

In $[\mathrm{Pd}]$, John Pardon defined a notion of an "implicit atlas" on a compact Hausdorff space, and used it to define a virtual fundamental class. After a very brief review of Pardon's setup, we establish a basic fact, Lemma 7.1 below, about his fundamental class. This lemma, and its rather technical proof, are entirely within Pardon's setup, and can be regarded as a small addendum to his paper [Pd]. Lemma 7.1 is used in Section 8 to relate Pardon's virtual fundamental class to the relative fundamental class.

We start with a brief review of Pardon's implicit atlas package [Pd, Definition 3.1.1]. Let $X$ be a compact Hausdorff space. Roughly speaking, an implicit atlas $\mathcal{A}$ on $X$ with index set $A$ organizes a collection of local charts indexed by finite subsets $I=\left\{\alpha_{1}, \ldots \alpha_{k}\right\}$ of $A$ (including $I=\emptyset$ ). Each chart consists of

(i) a "thickening" space $X_{I}$ containing an open subset $X_{I}^{r e g} \subseteq X_{I}$ that is a manifold,

(ii) an "obstruction space" $E_{I}=\oplus_{\alpha \in I} E_{\alpha}$ which is a vector space of dimension $\operatorname{dim} E_{I}=$ $\operatorname{dim} X_{I}^{r e g}-d$, and

(iii) "Kuranishi maps" $s_{\alpha}: X_{I} \rightarrow E_{\alpha}$ for each $\alpha \in I$.

One can also include finite groups $\Gamma_{\alpha}$ acting linearly on $E_{\alpha}$ for each $\alpha \in A$. There is additional data needed to ensure compatibility, e.g. "footprint" maps $\psi_{I J}$ defined for each $I \subseteq J \subseteq A$. These are required to satisfy a host of compatibility conditions and transversality axioms $[\mathrm{Pd}$, Definition 3.1.1]. In particular, the existence of an implicit atlas implies that $X$ is locally metrizable, and hence metrizable since $X$ is compact.

Assuming that the implicit atlas is locally orientable in the sense of [Pd, Definition 4.1.2], Pardon associates a virtual cochain complex [Pd, §4], and uses its homology to define a virtual fundamental class

$$
[X]_{A}^{\operatorname{vir}} \in \check{\mathrm{H}}^{d}\left(X ; \mathfrak{o}_{X} \text { rel } \partial\right)^{\vee}
$$

in the dual of rational Cech cohomology with coefficients in an orientation sheaf. Specifically, $[X]_{A}^{\mathrm{vir}}$ is defined in $\S 5.1$ of $[\mathrm{Pd}]$ as the composition

$$
\check{\mathrm{H}}^{d}\left(X ; \mathfrak{o}_{X \text { rel } \partial}\right)=H_{\text {vir }}^{d}(X \operatorname{rel} \partial ; A) \stackrel{s_{*}}{\longrightarrow} H_{0}(E, A) \stackrel{\left[E_{A}\right] \mapsto 1}{\longrightarrow} \mathbb{Q} .
$$


Pardon defines (7.1) in the general context of an implicit atlas with boundary. In the special case of implicit atlases, which suffices for our purposes, the sheaf $\mathfrak{o}_{X}$ rela could be written as $\mathfrak{o}_{X}$. Nevertheless, we will retain Pardon's "rel $\partial$ " notation to facilitate comparison with Sections 4 and 5 of [?].

In general, a space $X$ with an implicit atlas $A$ contains a distinguished open subset, the regular locus $X_{\emptyset}^{\text {reg }}$ of $X$, which is a $d$-dimensional manifold ( without boundary, but not necessarily compact). Along the regular locus, Pardon's virtual orientation sheaf $\mathfrak{o}_{X}$ rel $\partial$ is canonically identified with the orientation sheaf of $X_{\emptyset}^{r e g}$ as a manifold $[\mathrm{Pd}$, Definition 4.1.3].

The regular locus $X_{\emptyset}^{r e g}$ is an open subset of the compact set $X$, so the inclusion

$$
j: X_{\emptyset}^{r e g} \hookrightarrow X
$$

induces a map in compactly supported Čech cohomology, and hence a diagram

$$
\begin{array}{cc}
\check{\mathrm{H}}_{c}^{d}\left(X ; j ! j^{*} \mathfrak{o}_{X \text { rel } \partial}\right) & \rightarrow \check{\mathrm{H}}_{c}^{d}\left(X ; \mathfrak{o}_{X \text { rel } \partial}\right) \\
j ! \uparrow \cong & \downarrow= \\
\check{\mathrm{H}}_{c}^{d}\left(X_{\emptyset}^{r e g} ; \mathfrak{o}_{X \text { rel } \partial}\right) & \check{\mathrm{H}}^{d}\left(X ; \mathfrak{o}_{X \text { rel } \partial}\right)
\end{array}
$$

where the horizontal map is induced by the map of sheaves $j ! j^{*} \mathcal{F} \rightarrow \mathcal{F}$, and the vertical maps are as in Lemmas A.4.7 and A.4.5 of [ $[\mathrm{Pd}]$. The dual of the composition is a map

$$
\rho_{\text {reg }}: \check{\mathrm{H}}^{d}\left(X ; \mathfrak{o}_{X} \text { rel } \partial\right)^{\vee} \longrightarrow \check{\mathrm{H}}_{c}^{d}\left(X_{\emptyset}^{r e g} ; \mathfrak{o}_{X} \text { rel } \partial\right)^{\vee} .
$$

Now assume for simplicity that the regular locus $X_{\emptyset}^{r e g}$ is oriented. Then it carries a fundamental class in rational Steenrod homology

$$
\left[X_{\emptyset}^{r e g}\right] \in{ }^{s} \mathrm{H}_{d}\left(X_{\emptyset}^{r e g}, \mathbb{Q}\right) .
$$

The natural isomorphism $\tau$ defined by (A.12) takes this to the fundamental class

$$
\left[X_{\emptyset}^{r e g}\right] \in \check{\mathrm{H}}_{c}^{d}\left(X_{\emptyset}^{r e g} ; \mathbb{Q}\right)^{\vee}
$$

in the dual of compactly supported rational Cech cohomology. The orientation also determines an isomorphism

$$
\check{\mathrm{H}}_{c}^{d}\left(X_{\emptyset}^{r e g} ; \mathfrak{o}_{X \text { rel } \partial}\right)^{\vee} \stackrel{\cong}{\longrightarrow} \check{\mathrm{H}}_{c}^{d}\left(X_{\emptyset}^{r e g} ; \mathbb{Q}\right)^{\vee}
$$

In the special case that the regular locus is all of $X, X=X_{\emptyset}^{r e g}$ is a topological manifold, and Pardon shows that the class (7.1) determined by an orientable implicit atlas is equal to the usual fundamental class (7.5) [ $[\mathrm{Pd}$, Lemma 5.2.6]. For our purposes, we need the following more general fact, whose proof was communicated to us by Pardon. It asserts that, for any orientable implicit atlas, the restriction of (7.1) to the regular locus $X_{\emptyset}^{\text {reg }}$ is the fundamental class (7.5).

Lemma 7.1 (Pardon). Let $X$ be a compact Hausdorff space with a d-dimensional locally orientable implicit atlas $A$. Assume that the regular locus $X_{\emptyset}^{\text {reg }}$ is oriented. Then

$$
\rho_{\text {reg }}[X]_{A}^{\text {vir }}=\left[X_{\emptyset}^{\text {reg }}\right]
$$

where the righthand side corresponds to the fundamental class (7.5) under (7.6). 
Proof. The proof is an exercise understanding the naturality of the map (7.4) in Pardon's VFC package. Before starting, note that metrizable spaces are paracompact, and that we can assume that the atlas $A$ is finite (cf. Section 5.1 of $[\mathrm{Pd}]$ ).

In the context of Section 4.3 of $[\mathrm{Pd}]$, we have sets $V_{I}$ defined by $V_{I}=\psi_{\emptyset I}\left(s_{I}^{-1}(0) \cap X_{I}^{r e g}\right)$ associated to each subset $I$ of $A$. In the special case $I=\emptyset$, axioms (iv) and (vii) in the definition of the implicit atlas [Pd, Definition 3.1.2] imply that $s_{\emptyset}=0$ and $\psi_{\emptyset, \emptyset}=i d$, and hence $V_{\emptyset}=X_{\emptyset}^{r e g}$. Taking $K=X$ and $I=J=\emptyset$, we have

$$
H_{\text {vir }}^{d}(X \operatorname{rel} \partial, A)_{\emptyset \emptyset}=\check{\mathrm{H}}_{c}^{d}\left(X_{\emptyset}^{r e g} ; \mathfrak{o}_{X \text { rel } \partial}\right)
$$

by $\left[\mathrm{Pd}\right.$, (4.3.9)]. Furthermore, the map $j: V_{I} \cap V_{J} \rightarrow X$ used in Section 4.3 of [Pd] is equal to (7.2) in the case $I=J=\emptyset$, and hence

$$
H_{\text {vir }}^{d}(X \operatorname{rel} \partial, A)_{\emptyset \emptyset}=\check{\mathrm{H}}^{d}\left(X ; j ! j^{*} \mathfrak{o}_{X} \text { rel } \partial\right)
$$

by $[\mathrm{Pd},(4.3 .11)]$. We then have a commutative diagram

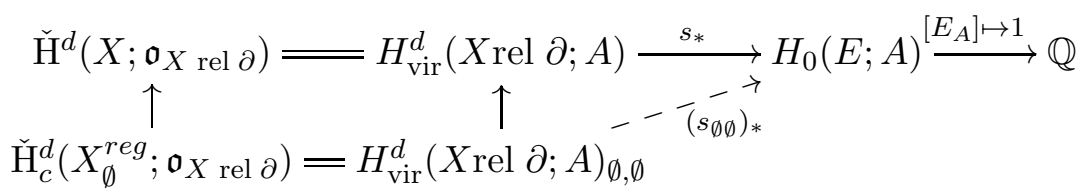

where the first vertical arrow is from (7.3), and the second vertical arrow is induced by the maps (4.2.8) and (4.2.10) in $[\mathrm{Pd}$. The square in (77.9) is commutative by the constructions in Section 4.3 of $[\mathrm{Pd}$ (the top isomorphism is the homotopy colimit of the isomorphisms (4.3.11), which include the case $I=J=\emptyset$ ). Diagram (7.9) shows that $\rho_{\text {reg }}[X]_{A}^{\text {vir }}$ is the composition

$$
\check{\mathrm{H}}_{c}^{d}\left(X_{\emptyset}^{r e g} ; \mathfrak{o}_{X \text { rel } \partial}\right)=H_{\mathrm{vir}}^{d}(X \mathrm{rel} \partial ; A)_{\emptyset, \emptyset} \stackrel{\left(s_{\emptyset \emptyset}\right)_{*}}{\rightarrow---H_{0}}(E, A) \stackrel{\left[E_{A}\right] \mapsto 1}{\longrightarrow} \mathbb{Q} .
$$

The proof is completed by showing that the composition (7.10) is the fundamental class (7.5). We do this by looking at the chain level, using (4.2.3), (4.2.4), (4.2.12) in [Pd] as follows. In the case $I=J=\emptyset$, Definition 4.2.1 of $[\mathrm{Pd}]$ reduces to

$$
X_{\emptyset \emptyset A}=E_{A} \times\{0\} \times X_{\emptyset}^{r e g},
$$

which we identify with $E_{A} \times X_{\emptyset}^{r e g}$. This is an orientable $d+\operatorname{dim} E_{A}$ dimensional manifold. The space $X_{I, J, A}$ in Definition 4.2.1 comes with a $\Gamma_{J}$ action, which is implicitly extended to an action of $\Gamma_{A}$ (by the canonical map $\Gamma_{A} \rightarrow \Gamma_{J}$ ). In particular, $\Gamma_{A}$ acts trivially on the factor $X_{\emptyset}^{r e g}$ of (7.11). Similarly, for each $K \subseteq X$, Definition 4.2.1 of [Pd] gives

$$
X_{\emptyset \emptyset A}^{K}=\{0\} \times\left(K \cap X_{\emptyset}^{r e g}\right),
$$

which we similarly identify with $K \cap X_{\emptyset}^{r e g}$. In particular, taking $K=X$ gives the identification

$$
C_{\operatorname{dim} E_{A}}\left(X_{\emptyset \emptyset A}, X_{\emptyset \emptyset A} \backslash X_{\emptyset}^{r e g}, \mathfrak{o}_{E_{A}}^{\vee}\right)^{\Gamma_{A}}=C_{\operatorname{dim} E_{A}}\left(E_{A} \times X_{\emptyset}^{r e g},\left(E_{A} \backslash 0\right) \times X_{\emptyset}^{r e g}, \mathfrak{o}_{E_{A}}^{\vee}\right)^{\Gamma_{A}} .
$$

Next, as in $[\mathrm{Pd},(4.2 .7),(4.2 .12)]$, the map $s_{\emptyset \emptyset *}$ is defined as the composition around the square

$$
\begin{gathered}
C_{\text {vir }}^{d}(X \operatorname{rel} \partial ; A)_{\emptyset, \emptyset} \frac{s_{\emptyset \emptyset *}}{\|} C_{0}(E, A) . \\
C_{\operatorname{dim} E_{A}}\left(X_{\emptyset \emptyset A}, X_{\emptyset \emptyset A} \backslash X_{\emptyset}^{r e g}, \mathfrak{o}_{E_{A}}^{\vee}\right)^{\Gamma_{A}} \rightarrow C_{\operatorname{dim} E_{A}}\left(E_{A}, E_{A} \backslash 0 ; \mathfrak{o}_{E_{A}}^{\vee}\right)^{\Gamma_{A}} .
\end{gathered}
$$


Here, the vertical arrows are the identifications (4.2.4) and (4.2.3) in [Pd], respectively, and the bottom map is induced by the projection $X_{\emptyset \emptyset A} \rightarrow E_{A}$ onto the first factor of (7.11). Passing to homology and using (7.8) gives a commutative diagram

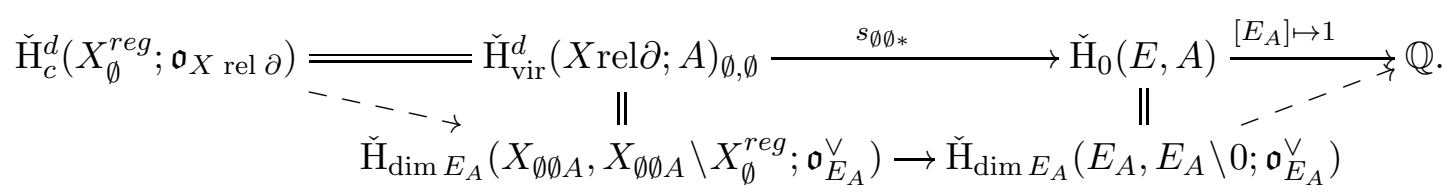

whose top row is (7.10). The first dashed arrow is Poincaré-Lefschetz duality in the form $[\mathrm{Pd}$, (A.6.6)] (Pardon's equations (4.3.3)-(4.3.11), used above to obtain (7.8), reduce to this for $I=J=\emptyset)$. Moreover, by (7.11) and Kunneth decomposition, the lower left group is $H_{\operatorname{dim} E_{A}}\left(E_{A}, E_{A} \backslash 0\right) \otimes H_{0}\left(X_{\emptyset}^{r e g}\right)$, and the homology map induces by $X_{\emptyset \emptyset A} \rightarrow E_{A}$ is id $\otimes \varepsilon$ where $\varepsilon: H_{0}\left(X_{\emptyset}^{r e g}\right) \rightarrow \mathbb{Q}$ is the augmentation. It follows that the composition

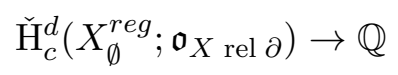

along the bottom of the diagram is equal to the fundamental class (7.5) after trivializing the orientation sheaf over $X^{\text {reg }}$.

\section{Relating Relative and Virtual Fundamental Classes}

In this final section, we consider spaces that are thin compactifications and admit an orientable implicit atlas. Such a space $\bar{M}$ is compact, metrizable, and has both the fundamental class $[\bar{M}]$ defined in Section 1, and the Pardon virtual fundamental class $[\bar{M}]_{A}^{\text {vir }}$ as in (7.1). We give conditions under which these correspond, first for a single compact space in Proposition 8.1, and then for families in Theorem 8.3.

We will work with rational coefficients, moving between Steenrod homology and dual Čech cohomology using the natural transformation

$$
\tau:{ }^{s} \mathrm{H}_{k}(X ; \mathbb{Q}) \stackrel{\cong}{\longrightarrow} \check{\mathrm{H}}_{c}^{k}(X ; \mathbb{Q})^{\vee}
$$

(see (A.12) ) which is an isomorphism for any paracompact, locally compact space $X$. Furthermore, if $X$ admits an orientable implicit atlas $A$, an orientation of $A$ (i.e. a nowhere vanishing section of $\mathfrak{o}_{X}$ rel $\partial$ ) trivializes the orientation sheave, giving an identification

$$
\check{\mathrm{H}}^{k}\left(X ; \mathfrak{o}_{X} \text { rel } \partial\right)^{\vee}=\check{\mathrm{H}}^{k}(X ; \mathbb{Q})^{\vee} .
$$

The orientation on $A$ also orients the regular locus $\bar{M}_{\emptyset}^{\text {reg }}$.

Proposition 8.1. Let $M$ be an oriented manifold. If a thin compactification $\bar{M}$ of $M$ admits an oriented implicit atlas $A$ such that $M \subseteq \bar{M}_{\emptyset}^{\mathrm{reg}}$ as oriented manifolds of same dimension $d$, then the fundamental class (1.6) corresponds to Pardon's virtual fundamental class:

$$
[\bar{M}]=[\bar{M}]_{A}^{\operatorname{vir}}
$$

under the isomorphisms (8.1) and (8.2) for $X=\bar{M}$.

Proof. In this situation, there are inclusions $M \subseteq \bar{M}_{\emptyset}^{\text {reg }} \subseteq \bar{M}$ of open subsets, where $M$ and $\bar{M}_{\emptyset}^{r e g}$ are oriented paracompact $d$-manifolds, and $\bar{M}$ is compact. The corresponding Steenrod 
restriction maps (1.1) induce a commutative diagram

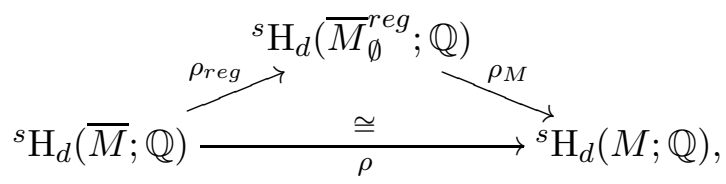

where the bottom arrow is the isomorphism (1.5). This maps via (8.1) to a corresponding diagram in the dual of rational Čech cohomology, in which $\rho_{\text {reg }}$ is replaced by the Čech map

$$
\rho_{\text {reg }}: \check{\mathrm{H}}^{d}(\bar{M} ; \mathbb{Q})^{\vee} \longrightarrow \check{\mathrm{H}}_{c}^{d}\left(\bar{M}_{\emptyset}^{r e g} ; \mathbb{Q}\right)^{\vee},
$$

induced by the inclusion $\bar{M}_{\emptyset}^{r e g} \hookrightarrow \bar{M}$, and thus corresponding to (7.4) with $X=\bar{M}$ under the coefficient trivialization (8.2). With these identifications, the statement of Lemma 7.1 becomes

$$
\rho_{\text {reg }}[\bar{M}]_{A}^{\mathrm{vir}}=\left[\bar{M}_{\emptyset}^{r e g}\right] \quad \text { in } \check{\mathrm{H}}_{c}^{d}\left(\bar{M}_{\emptyset}^{r e g} ; \mathbb{Q}\right)^{\vee} .
$$

Successively applying equations (1.6), (1.3) and (8.3), and then using the commutativity of the diagram, we obtain

$$
\rho[\bar{M}]=[M]=\rho_{M}\left[\bar{M}_{\emptyset}^{r e g}\right]=\rho_{M}\left(\rho_{\text {reg }}[\bar{M}]_{A}^{\mathrm{vir}}\right)=\rho[\bar{M}]_{A}^{\mathrm{vir}} .
$$

The proposition follows because $\rho$ is an isomorphism.

For applications, one needs a version of Proposition 8.1 for families. While Pardon does not explicitly describe implicit atlases for families, his applications (e.g. [Pd, §9.3]) show that it is reasonable to consider proper continuous maps

$$
\underset{\mathcal{P}}{\mathcal{X}}
$$

from a Hausdorff space to a Banach manifold that satisfy two conditions:

IA.1. Every fiber $\mathcal{X}_{p}$ of (8.4) admits an oriented implicit atlas $A_{p}$.

IA.2. For every path $\gamma$ in $\mathcal{P}$ from $p$ to $q$, there is an oriented implicit atlas with boundary on $\mathcal{X}_{\gamma}$ which restricts to the chosen oriented implicit atlas on $\mathcal{X}_{p}$ and $\mathcal{X}_{q}$.

By (IA.1), each fiber has a virtual fundamental class

$$
\left[\mathcal{X}_{p}\right]_{A_{p}}^{\operatorname{vir}} \in \check{\mathrm{H}}^{d}\left(\mathcal{X}_{p} ; \mathbb{Q}\right)^{\vee}
$$

while (IA.2) implies that

$$
\left[\mathcal{X}_{p}\right]_{A_{p}}^{\mathrm{vir}}=\left[\mathcal{X}_{q}\right]_{A_{q}}^{\mathrm{vir}} \quad \text { in } \quad \check{\mathrm{H}}^{d}\left(\mathcal{X}_{\gamma} ; \mathbb{Q}\right)^{\vee}
$$

(cf. the proof of Lemma 9.3.2 of [Pd] ). Applying Proposition 1.3 we obtain:

Lemma 8.2. For a family (8.4) that satisfies conditions (IA.1) and (IA.2), the association $p \mapsto\left[\mathcal{X}_{p}\right]_{A_{p}}^{\mathrm{vir}}$ extends uniquely to a relative homology functor $\mu_{A}^{\mathrm{vir}}$. 
To extend Proposition 8.1 to families, we again consider a relatively oriented Fredholm family (2.1) that admits a metrizable, relatively thin compactification

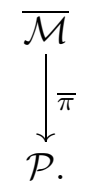

We also assume that $\overline{\mathcal{M}}$ satisfies (IA.1) and (IA.2) above, with an inclusion

$$
\mathcal{M}_{p} \subseteq\left(\overline{\mathcal{M}}_{p}\right)_{\emptyset}^{r e g} \quad \text { for all } p \in \mathcal{P} .
$$

In this case, both the relative fundamental class $[\overline{\mathcal{M}}]^{r f c}$ and Pardon's virtual fundamental class $[\overline{\mathcal{M}}]_{A}^{\text {vir }}$ are defined, and both are relative homology functors. The following theorem shows that they are equal.

Theorem 8.3. Suppose that a metrizable, relatively thin compactification $\bar{\pi}: \overline{\mathcal{M}} \rightarrow \mathcal{P}$ of a relatively oriented Fredholm family satisfies (IA.1), (IA.2) and (8.6). Then the relative fundamental class and Pardon's virtual fundamental class are equal as relative homology functors. In particular,

$$
\left[\overline{\mathcal{M}}_{p}\right]^{r f c}=\left[\overline{\mathcal{M}}_{p}\right]_{A_{p}}^{\operatorname{vir}}
$$

for all $p \in \mathcal{P}$.

Proof. For each regular value $p$ of $\bar{\pi}, \overline{\mathcal{M}}_{p}$ is a metrizable, relatively thin compactification of $\mathcal{M}_{p}$, and has an implicit atlas $A_{p}$ with $\mathcal{M}_{p} \subset\left(\overline{\mathcal{M}}_{p}\right)_{\emptyset}^{r e g}$. Proposition 8.1 then shows that $\left[\overline{\mathcal{M}}_{p}\right]^{r f c}=\left[\overline{\mathcal{M}}_{p}\right]=\left[\overline{\mathcal{M}}_{p}\right]_{A_{p}}^{\text {vir }}$. But the set of regular values of $\pi$ is dense in $\mathcal{P}$, so Proposition 1.3(a) gives (8.7).

Corollary 8.4. Assume $\bar{\pi}: \overline{\mathcal{M}} \rightarrow \mathcal{P}$ satisfies the assumptions of Theorem 8.3 and $\bar{f}: \overline{\mathcal{M}} \rightarrow$ $Y$ a continuous map. Then for each class $\beta \in \check{\mathrm{H}}^{d}(Y ; \mathbb{Q})$ the function

$$
I_{\beta}(p)=\left\langle f_{*}\left[\overline{\mathcal{M}}_{p}\right]^{r f c}, \beta\right\rangle=\left\langle f_{*}[\overline{\mathcal{M}}]_{A_{p}}^{\text {vir }}, \beta\right\rangle
$$

is independent of $p$ on each path-connected component of $\mathcal{P}$.

Proof. Because each fiber $\overline{\mathcal{M}}_{p}$ is compact, the restriction of $f$ to $\overline{\mathcal{M}}_{p}$ is proper and continuous, so induces a map $f_{*}$ in Čech homology. Then (8.8) follows from (8.7) and (1.23).

\section{Appendix A. COMPARISON OF HOMOlogy theORIES}

This first appendix records needed facts about the Steenrod, Čech and Borel-Moore homology and the corresponding cohomology theories, and provides references.

A.1. Spaces. We will consider five categories: the category $\mathcal{A}$ of Hausdorff spaces and continuous maps, the subcategories $\mathcal{A}_{C}$ of compact spaces and $\mathcal{A}_{C M}$ of compact metric spaces, the category $\mathcal{A}_{L C}$ of locally compact spaces and proper continuous maps, and the subcategory $\mathcal{A}_{E C} \subset \mathcal{A}_{L C}$ of locally compact, separable metric spaces with finite (covering) dimension. Every $n$-dimensional separable metric space is homeomorphic to a subset of $\mathbb{R}^{2 n+1}[\mathrm{HW}$, Theorem V 3], and using local compactness one can lift this to a homeomorphism into $\mathbb{R}^{2 n+2}$ with a closed image [D, IV.8.2 and 8.3]. Thus objects in $\mathcal{A}_{E C}$ can be regarded as closed subsets $X$ of euclidean space ("euclidean closed"). 
For finite-dimensional Hausdorff manifolds, the properties of being $\sigma$-compact and second countable are equivalent, and any manifold with these properties is separable, paracompact, metrizable, and in the category $\mathcal{A}_{E C}$.

A.2. Homology. Steenrod homology ${ }^{s} \mathrm{H}_{*}$ and $\check{C}$ ech homology $\check{\mathrm{H}}_{*}$ are introduced in Section 1. For the intersection theory done in Sections 4 and 5, it is useful to also use Borel-Moore homology $H_{*}^{B M}$. Throughout, we restrict attention to constant coefficients in $R=\mathbb{Z}$ or $\mathbb{Q}$.

- Steenrod (also called Steenrod-Sitnikov) homology is defined on $\mathcal{A}_{L C}$ using chains that are dual to compactly supported, finite-value Alexander-Spanier cochains [Mas, §4]. It can also be defined on locally compact metrizable spaces using "canonical coverings" [Sk, Sk2]; see also Milnor's construction on compact pairs [Mil]. It has an extension to paracompact spaces in $\mathcal{A}$ called strong homology [Mar, Chapter 19].

- Čech homology is defined for pairs in $\mathcal{A}$ using nerves of covers. It is only a partially exact homology theory, but it has the Continuity Property (1.9) [ES, pages 260-261] and is exact for finitely triangulable spaces [ES, §IX.9].

- Borel-Moore homology $H_{*}^{B M}$ is defined on $\mathcal{A}_{L C}$ using sheaves [BM, $\mathrm{Br} 2, \mathrm{Iv}$ ] or on $\mathcal{A}_{E C}$ using singular cohomology [F1, Chapter 19], [F2, Appendix B]. It has all of the properties listed in Section 1 for Steenrod homology. In particular, for each open set $U \subseteq X$ there is a natural restriction map

$$
\rho_{U}: H_{*}^{B M}(X) \rightarrow H_{*}^{B M}(U)
$$

corresponding to (1.1) and an exact sequence corresponding to (1.2) (cf. [Iv, IX.2.1]).

Steenrod and Borel-Moore satisfy the Eilenberg-Steenrod axioms, so are naturally isomorphic to singular homology on the category of triangulable spaces. Both are single space theories in the sense of [ES, §X.7] (see page vii in [Mas] and Corollary V.5.10 and the cautionary note V.5.12 [Br2]). In particular, the theorems in Section X.7 of [ES] show that

$$
{ }^{s} \mathrm{H}_{*}(X, A)={ }^{s} \mathrm{H}_{*}(X \backslash A), \quad H_{*}^{B M}(X, A)=H_{*}^{B M}(X \backslash A)
$$

for any closed pair $(X, A)$. Steenrod and Borel-Moore homologies satisfy Minor's modified continuity property: if $\left\{X_{\alpha}\right\}$ is an inverse system in $\mathcal{A}_{C M}$ with $X=\lim X_{\alpha}$, then there is a natural exact sequence

$$
0 \longrightarrow \lim ^{1}{ }^{s} \mathrm{H}_{k+1}\left(X_{\alpha}\right) \longrightarrow{ }^{s} \mathrm{H}_{k}(X) \longrightarrow \lim ^{s} \mathrm{H}_{k}\left(X_{\alpha}\right) \longrightarrow 0,
$$

and a corresponding sequence with ${ }^{s} \mathrm{H}_{*}$ replaced by $H_{*}^{B M}$ [Mil, Thm. 4], [Mas2, p.81], [Br2, V.5.15]. There are many axiomatic characterizations of Steenrod homology (cf. [In, BeM]. In particular, Theorem 1 of [In] asserts:

- Up to natural isomorphism, ${ }^{s} \mathrm{H}_{*}(X ; G)$ is the unique homology theory on $\mathcal{A}_{C}$ that satisfies the Eilenberg-Steenrod axioms, is functorial in both $X$ and $G$, and has the continuity property (1.9) for infinitely divisible abelian groups $G$.

A.3. Natural Transformations. For any closed subset $X$ of a paracompact $n$-dimensional oriented manifold $N$ and $R=\mathbb{Z}$ or $\mathbb{Q}$, there is a natural isomorphism (a version of Steenrod duality)

$$
H^{k}(N, N \backslash X ; R) \stackrel{\cong}{\longrightarrow} \mathrm{H}_{n-k}(X ; R)
$$

(see [Mas, Theorem 11.15], using the isomorphism from the Alexander-Spanier to the singular cohomology of $(N, N \backslash X)$ in the proof of [Sp1, Cor. 6.9.6]). There is a similar isomorphism 
(Poincaré-Alexander duality)

$$
H^{k}(N, N \backslash X ; R) \stackrel{\cong}{\longrightarrow} H_{n-k}^{B M}(X ; R)
$$

for Borel-Moore homology [Sp2, Thm. 10.4] or [IV, IX.4.7]. Taking $N=\mathbb{R}^{n}$, (A.3) and (A.4) together show that there is a natural isomorphism $\beta$ from Steenrod to Borel-Moore homology on the category $\mathcal{A}_{E C}$. In fact, on $\mathcal{A}_{E C}$ with $R=\mathbb{Z}$ or $\mathbb{Q}$, there is a commutative diagram of natural transformations

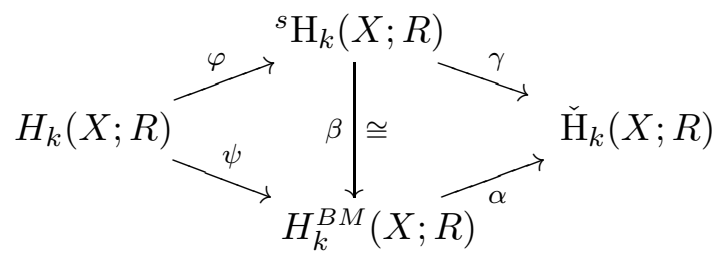

where $\gamma$ is as in [Sk, Theorem 4], $\psi=\beta \circ \varphi$ and $\alpha=\gamma \circ \beta^{-1}$ ( $\varphi$ factors through compactly supported homology: see page 291 and the first arrow on page 308 in [Mas.) Furthermore,

(i) The isomorphism $\beta$ carries the restriction map (1.1) to the corresponding restriction map in Borel-Moore homology, and carries the exact sequence (1.2) to the corresponding Borel-Moore sequence by matching both to the exact sequence

$$
\cdots \longrightarrow H^{k-1}(N \backslash X) \stackrel{\delta}{\longrightarrow} H^{k}(N, N \backslash X) \stackrel{j^{*}}{\longrightarrow} H^{k}(N) \stackrel{\iota^{*}}{\longrightarrow} H^{k}(N \backslash X) \longrightarrow \cdots
$$

in Alexander-Spanier (or equivalently singular) cohomology; cf. Theorems 11.15 and 8.1 in [Mas], and diagram IX.3.5 in [Iv]. (Steenrod and Borel-Moore homologies are also isomorphic on the category $\mathcal{A}_{C M}[\mathrm{BM}, \S 5]$.)

(ii) $\gamma$ is surjective, and is an isomorphism if $R=\mathbb{Q}$, or if $R=\mathbb{Z}$ and either (i) $k=\operatorname{dim} X$ or (ii) $X$ is a compact and locally contractible [Sk, Thm. 4].

(iii) $\varphi$ and $\psi$ are isomorphisms if $X$ is compact and locally contractible [Mas, §9.6], [Br2, $\S \mathrm{V} .12]$.

(iv) $\alpha$ is an isomorphism for locally contractible spaces $X$ in $\mathcal{A}_{C M}$ [Br2, V.5.19].

In particular, the natural transformations in (A.5) give isomorphisms

$$
H_{*}^{B M}(X ; \mathbb{Q}) \cong{ }^{s} \mathrm{H}_{*}(X ; \mathbb{Q}) \cong \check{\mathrm{H}}_{*}(X ; \mathbb{Q})
$$

for compact metric spaces $X$, and

$$
H_{*}(N ; \mathbb{Z}) \stackrel{\varphi}{\cong}{ }^{s} \mathrm{H}_{*}(N ; \mathbb{Z}) \stackrel{\gamma}{\cong} \check{\mathrm{H}}_{*}(N ; \mathbb{Z})
$$

for compact manifolds $N$ and for finite polyhedra.

A.4. Cohomology. Singular, Čech and Alexander-Spanier cohomology are all defined on the category of pairs in $\mathcal{A}$; we will restrict attention to paracompact pairs and constant coefficients $R=\mathbb{Z}$ or $\mathbb{Q}$. The last two are naturally isomorphic [Sp1, 6.8.8 and Exercise 6.D.3], so we use the notation $\check{\mathrm{H}}^{*}$ for both. The corresponding theories based on compactly supported cochains are also isomorphic, and will be denoted by $\check{\mathrm{H}}_{c}^{*}$. In Part II of [Mas], Massey also uses locally finite-valued cochains for closed pairs, but these give the same theory as Alexander-Spanier cohomology by [Mas, Thm. 8.1]. Thus for paracompact pairs $(X, A)$ and $R=\mathbb{Z}$ or $\mathbb{Q}$, it suffices to consider three theories: $\check{\mathrm{H}}^{*}, \check{\mathrm{H}}_{c}^{*}$ and singular cohomology $H^{*}$. These are related by natural transformations

$$
\check{\mathrm{H}}_{c}^{*}(X, A ; R) \stackrel{\mu}{\longrightarrow} \check{\mathrm{H}}^{*}(X, A ; R) \stackrel{\nu}{\longrightarrow} H^{*}(X, A ; R)
$$


which preserve cup products [Br2, Thm. III.2.1 and Cor. III.4.12] and [Sp1, §6.5, §6.9]. Furthermore,

(i) $\mu$ is an isomorphism if $X$ is compact [Sp1, 6.6.9].

(ii) $\nu$ is an isomorphism if $X$ and $A$ are locally contractible (e.g. manifolds) and $A$ is either closed [Br2, Theorem III.2.1] or open (proof of 6.9.6 in [Sp1]).

For paracompact, locally compact spaces $X$, Steenrod homology pairs with $\check{H}_{c}^{*}$ in the sense that there is a Kronecker pairing

$$
{ }^{s} \mathrm{H}_{*}(X) \otimes \check{\mathrm{H}}_{c}^{*}(X) \rightarrow R
$$

(cf. [Mas, §4.8]), and an exact sequence

$$
0 \rightarrow \operatorname{Ext}\left(\check{\mathrm{H}}_{c}^{k+1}(X), R\right) \longrightarrow{ }^{s} \mathrm{H}_{k}(X ; R) \stackrel{\tau}{\longrightarrow} \operatorname{Hom}\left(\check{\mathrm{H}}_{c}^{k}(X), R\right) \rightarrow 0
$$

that is natural in both $X$ and $R$ [Mas, Cor. 4.18 and p.371]. The same sequence holds with ${ }^{s} \mathrm{H}_{*}$ replaced by $H_{*}^{B M}[\mathrm{BM}$, Theorem 3.3]. In particular, there is a natural transformation

$$
\tau:{ }^{s} \mathrm{H}_{k}(X ; R) \longrightarrow \check{\mathrm{H}}_{c}^{k}(X ; R)^{\vee}
$$

where ${ }^{\vee}$ denotes dual, i.e. $\operatorname{Hom}(\cdot, R)$. By (A.11), $\tau$ is an isomorphism for $R=\mathbb{Q}$. For compact metric spaces, it factors through Cech homology, as follows.

Lemma A.1. On the category $\mathcal{A}_{C M}$ with $R=\mathbb{Z}$ or $\mathbb{Q}, \check{\mathrm{H}}_{c}^{*}(X)=\check{\mathrm{H}}^{*}(X)$ and there is a commutative diagram of natural transformations

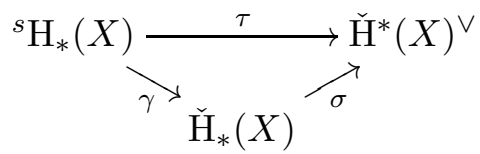

with $\gamma$ as in (A.5) and $\sigma$ as defined below. For $R=\mathbb{Q}$, all three maps are isomorphisms.

Proof. First note that the compact metric space $X$ can be written as the inverse limit of a system $\left\{X_{\ell}\right\}$ in the category of finite polyhedra (cf. [Mas2, p.82] and [Sp1, 6.6.7]). The homology maps induced by the inclusions $X \rightarrow X_{\ell}$ define maps $\iota_{*}, j_{*}$ and $\check{\iota}_{*}$ into inverse systems as in the diagram below. But $\gamma$ is an isomorphism for finite polyhedra, thus we get a diagram (A.13) when $X$ is replaced by $X_{\ell}$, and $\sigma$ is defined to be $\tau \circ \gamma^{-1}$. By the naturality of $\gamma$ and $\tau$, there are induced maps $\gamma_{\bullet}, \tau_{\bullet}$ and $\sigma_{\bullet}$ between the inverse systems, and the front left and back squares in the diagram commute. Furthermore, $\gamma_{\bullet}$ is an isomorphism, and the bottom triangle commutes.

The map $\check{\iota}_{*}$ is an isomorphism by Čech continuity, and $j_{*}$ is an isomorphism by the continuity of Cech cohomology and the fact that $\underset{\longleftarrow}{\lim } \operatorname{Hom}(\cdot, R)=\operatorname{Hom}(\lim \cdot R)$. The first statement of the lemma follows by defining $\sigma$ to be the composition $\check{\iota}_{*}^{-1} \sigma_{\bullet} j_{*}$.

For $R=\mathbb{Q}$, the $\lim ^{1}$ term in $(\mathrm{A} .2$ ) and the Ext term in A.11 vanish. Hence $\iota_{*}$ and $\tau$, and therefore $\gamma$ and $\sigma$, are isomorphisms (cf. $\mathrm{Pd}$,

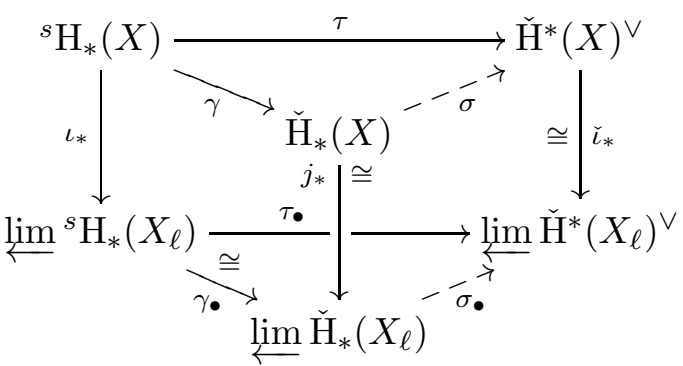
Remark 5.0.2]). 
Thus on $\mathcal{A}_{C M}, \sigma$ defines a Kronecker pairing $\check{\mathrm{H}}_{*}(X) \otimes \check{\mathrm{H}}^{*}(X) \rightarrow R$ in Čech theory, and this is related to (A.10) by

$$
\langle\gamma(b), \alpha\rangle=\langle b, \alpha\rangle \quad \forall b \in{ }^{s} \mathrm{H}_{*}(X), \alpha \in \check{\mathrm{H}}^{*}(X) .
$$

A.5. Borel-Moore via embeddings. Fulton and MacPherson developed a simplified version of Borel-Moore homology on $\mathcal{A}_{E C}$ for use in algebraic geometry; see Chapter 19 of [F1] or Appendix B of [F2]. Fixing coefficients in a field or in $\mathbb{Z}$, one defines the embedded Borel-Moore homology of a closed subset $X$ of $\mathbb{R}^{m}$ by formula (A.4):

$$
\bar{H}_{k}(X) \stackrel{\text { def }}{=} H^{m-k}\left(\mathbb{R}^{m}, \mathbb{R}^{m} \backslash X\right),
$$

(cf. [F1, §19.1, eq (1)]). Similarly, for any open subset $U$ of $X$, one sets

$$
\bar{H}_{k}(U)=\bar{H}_{k}(X, A)=H^{m-k}\left(\mathbb{R}^{m} \backslash A, \mathbb{R}^{m} \backslash X\right) .
$$

where $A=X \backslash U$ (cf. [F2, (30) in Appendix B]). Using facts about singular cohomology, one can verify that the groups $\bar{H}_{*}$ have all of the properties of Borel-Moore homology (cf. [F1], Section 19.1 and especially Example 19.1.1). For a direct correspondence, note that for an object $X$ in $\mathcal{A}_{E C}$, the choice of a closed embedding $\iota: X \rightarrow \mathbb{R}^{m}$ with image $X^{e}$ induces natural isomorphisms

$$
H_{*}^{B M}(X) \stackrel{\cong}{\stackrel{\imath}{\iota}} H_{*}^{B M}\left(X^{e}\right) \cong \bar{H}_{*}\left(X^{e}\right)
$$

where the second is the composition of (A.4) and (A.15).

A.6. Cap Products. For any closed subset $X$ of a locally compact $Z$, we have a localized (sheaf theoretic supported) cap product

$$
H_{n}^{B M}(Z) \otimes H^{k}(Z, Z \backslash X) \stackrel{\cap}{\rightarrow} H_{n-k}^{B M}(X),
$$

on Borel-Moore homology with coefficients in any commutative Noetherian ring, cf. [Iv, IX.3.1, §IX.5 and §II.9], [F1, §19.1]. This has several naturality properties (cf. [Iv, §IX.3]), including:

- for a proper map $f:\left(Z^{\prime}, X^{\prime}\right) \rightarrow(Z, X)$ of closed, locally compact pairs,

$$
f_{*}\left(a^{\prime} \cap f^{*} \xi\right)=f_{*} a^{\prime} \cap \xi .
$$

- for closed subsets $X \stackrel{i}{\hookrightarrow} Z$ and $Y \subseteq Z$,

$$
(a \cap \xi) \cap i^{*} \eta=a \cap(\xi \cup \eta) \quad \text { in } H_{*}^{B M}(X \cap Y) .
$$

- if $X \subseteq Z$ is closed and $U \stackrel{j}{\hookrightarrow} Z$ is open, then the restriction to $U$ satisfies

$$
\rho_{U \cap X}(a \cap \xi)=\rho_{U}(a) \cap j^{*} \xi \quad \text { in } H_{*}^{B M}(U \cap X) .
$$

These hold for all $a \in H_{*}^{B M}(Z), a^{\prime} \in H_{*}^{B M}\left(Z^{\prime}\right), \xi \in H^{*}(Z, Z \backslash X)$ and $\eta \in H^{*}(Z, Z \backslash Y)$. 
A.7. Fundamental classes. Let $N$ be an oriented topological $n$-manifold (a Hausdorff space locally homeomorphic to $\mathbb{R}^{n}$ ). Its orientation determines fundamental classes

$$
[N] \in{ }^{s} \mathrm{H}_{n}(N ; \mathbb{Z}) \quad[N] \in H_{n}^{B M}(N ; \mathbb{Z})
$$

in Steenrod [Mas, §4.9] and Borel-Moore homology [Iv, IX.4.6]. The restriction of $[N]$ to an open set $U \subseteq N$ is the fundamental class of $U$ :

$$
\rho_{U}[N]=[U] .
$$

On each component $N_{\alpha}$ of $N$, the orientation determines isomorphisms ${ }^{s} \mathrm{H}_{n}\left(N_{\alpha} ; \mathbb{Z}\right)=\mathbb{Z}=$ $H_{n}^{B M}\left(N_{\alpha} ; \mathbb{Z}\right)$ under which $\left[N_{\alpha}\right]$ corresponds to 1 . The naturality of the transformation $\beta$ in (A.5) with respect to restriction maps then implies that the two fundamental classes (A.21) correspond under $\beta$.

For any closed subset $Y$ of $N$, the cap product (A.17) with the fundamental class is an isomorphism

$$
D: H^{k}(N, N \backslash Y) \stackrel{[N] \cap}{\cong} H_{n-k}^{B M}(Y)
$$

which is precisely (A.4) cf. IV, IX.4.7]. There is corresponding isomorphism with values in Steenrod homology. In particular, if $N$ is compact, taking $Y=N$ gives the Poincaré duality isomorphisms

$$
D: H^{k}(N) \stackrel{\cong}{\longrightarrow} H_{n-k}^{B M}(N) \quad D: H^{k}(N) \stackrel{\cong}{\longrightarrow} \mathrm{H}_{n-k}(N) .
$$

A.8. Submanifolds. Suppose that $N$ is a paracompact oriented $C^{1} n$-manifold and

$$
V \hookrightarrow N
$$

is a properly embedded oriented submanifold of codimension $k$. By identifying a neighborhood $U$ of $V$ in $N$ with the total space of the normal bundle to $V$ and using excision, the Thom class of the normal bundle defines a singular cohomology class

$$
u=u_{V, N} \in H^{k}(U, U \backslash V)=H^{k}(N, N \backslash V)
$$

Following Fulton [F1, §19.2], we call $u$ the orientation class of $V$ in $N$. Then $[V]$ and $[N]$ are related

$$
[V]=[N] \cap u_{V, N} \quad \text { in } H_{n-k}^{B M}(V)
$$

as in [IV, IX.4.9]. In particular, $[V]$ corresponds to $u_{V, N}$ under the duality (A.22). The naturality of Thom class gives a naturality property of $u$ : if a $C^{1}$ map $f: M \rightarrow N$ of oriented manifolds is transverse to $V$, then $W=f^{-1}(V)$ is an oriented submanifold of $M$ with orientation class

$$
u_{W, M}=f^{*} u_{V, N}
$$

A.9. Intersection Pairing. For closed subsets $X$ and $Y$ of a manifold $N$ as in $\S$ A.8, there is a cup product in singular cohomology

$$
H^{n-k}(N, N \backslash X) \otimes H^{n-\ell}(N, N \backslash Y) \stackrel{\cup}{\longrightarrow} H^{2 n-k-\ell}(N, N \backslash(X \cap Y))
$$

(cf. [Sp1, §5.6], noting that $\{N-X, N-Y\}$ is an excisive pair by [Sp1, 4.6.4]). The duality (A.22) translates the cup product into the cap product

$$
H_{k}^{B M}(X) \otimes H^{n-\ell}(N, N \backslash Y) \stackrel{\cap}{\longrightarrow} H_{k+\ell-n}^{B M}(X \cap Y)
$$


by the formula:

$$
b \cap \alpha=D\left(D^{-1} b \cup \alpha\right) .
$$

Note that the righthand side is $b \cap i^{*} \alpha$ for the cap product (A.17), where $i$ is the inclusion of $X$ into $N$ (cf. A.19) with $a=[N], b=D \xi=[N] \cap \xi$, and $\eta=i^{*} \alpha$ ). Thus this cap product depends only on the restriction of $\alpha$ to $X$.

Applying (A.22) again yields a natural intersection pairing in Borel-Moore homology

$$
H_{k}^{B M}(X) \otimes H_{\ell}^{B M}(Y) \stackrel{\bullet}{\longrightarrow} H_{k+\ell-n}^{B M}(X \cap Y)
$$

given by

$$
a \bullet b=D\left(D^{-1} b \cup D^{-1} a\right)=b \cap D^{-1} a .
$$

(The order reversal is needed to obtain the correct signs, cf. [Br2, §V.11]).

The intersection pairing (A.28) is natural with respect to the restriction map $\rho_{U}$ to any open subset $U$ of $N$ : the naturality of the cup product and (A.1) translates into the identity

$$
\rho_{U}(a \bullet b)=\rho_{U}(a) \bullet \rho_{U}(b) .
$$

In particular, if $U$ is any neighborhood of $X \cap Y$, then the restriction to $U$ induces the identity on $H_{*}^{B M}(X \cap Y)$ and hence

$$
a \bullet b=\rho_{U}(a) \bullet \rho_{U}(b) .
$$

Thus the intersection localizes on any open neighborhood of $X \cap Y$.

Example A.2. Let $X$ and $Y$ be properly embedded oriented submanifolds of an oriented $C^{1}$ manifold $N$. If $X$ and $Y$ intersect transversally, then $X \cap Y$ is an oriented manifold, and

$$
[X] \bullet[Y]=[X \cap Y] \quad \text { in } H_{*}^{B M}(X \cap Y) .
$$

The proof exactly as in the proof of Theorem VI-11.9 of Br1, using formulas A.19, (A.25), (A.29) and the naturality of Thom classes, and interpreting all terms as elements of BorelMoore homology.

More generally, consider proper maps $f: M \rightarrow N$ and $g: P \rightarrow N$ between oriented manifolds. Set

$$
Z=\{(x, y) \in N \times N \mid f(x)=g(y)\}
$$

and define $h: Z \rightarrow N$ by $h(x, y)=f(x)=g(y)$.

Lemma A.3. Suppose that maps $f$ and $g$ as above are transverse and have complementary dimensions in $N$. Then $Z$ is a 0-dimensional manifold, and has an induced orientation such that

$$
f_{*}[M] \bullet g_{*}[P]=h_{*}[Z]
$$

in $H_{0}^{B M}(X \cap Y ; \mathbb{Z})$, where $X$ and $Y$ are the images of $f$ and $g$.

Proof. The assumptions that $f$ and $g$ are transverse with complementary dimension imply that $Z$ is a discrete set of points and that $f, g$, and $f \times g$ are immersions at each point $z=(x, y) \in Z$. Hence we can find disjoint open neighborhoods $U_{p}$ of the points $p \in X \cap Y$ so that, for $U=\bigsqcup U_{p}$, we have

$$
f^{-1}(U)=\bigsqcup_{x \in f^{-1}(X \cap Y)} V_{x}, \quad \text { and } \quad g^{-1}(U)=\bigsqcup_{y \in g^{-1}(X \cap Y)} W_{y}
$$


where $\left\{V_{x}\right\}$ (resp. $\left\{W_{y}\right\}$ ) are disjoint neighborhoods of $x$ in $M$ (resp. $y$ in $P$ ), and where $f$ and $g$ restrict to a proper embeddings $f: V_{x} \rightarrow U$ and $g: W_{y} \rightarrow U$.

As in (A.31), the lefthand side of (A.33) localizes, and hence is a locally finite sum of local intersections

$$
f_{*}[M] \bullet g_{*}[P]=\sum_{(x, y) \in Z} f_{*}\left[V_{x}\right] \bullet g_{*}\left[W_{y}\right]=\sum_{(x, y) \in Z}\left[f\left(V_{x}\right) \cap g\left(W_{y}\right)\right],
$$

where the second equality is obtained by applying Example A.2. But $f\left(V_{x}\right) \cap g\left(W_{y}\right)$ is exactly $h(z)$, and is oriented as the local intersection of $f$ and $g$. This identification induces an orientation $[z] \in H_{0}^{B M}(z ; \mathbb{Z})$ for each $z \in Z$, and hence an orientation on $Z$. The lemma follows.

A.10. Intersection Numbers. With constant coefficients $R=\mathbb{Z}$ or $\mathbb{Q}$, the intersection of classes $a \in H_{k}^{B M}(X ; R)$ and $b \in H_{\ell}^{B M}(Y ; R)$ of complementary dimension (i.e. $k+\ell=n=$ $\operatorname{dim} N$ ) is a 0 -dimensional class

$$
a \bullet b \in H_{0}^{B M}(X \cap Y ; R) .
$$

If $X \cap Y$ is compact, there is an augmentation map $\varepsilon: H_{*}^{B M}(X \cap Y ; R) \rightarrow R$ (induced by the map from $X \cap Y$ to a point), and the intersection number is defined by

$$
a \cdot b=\varepsilon(a \bullet b) \in R .
$$

This can be written in many other ways:

$$
a \cdot b=\varepsilon(b \cap \alpha)=\langle b \cap \alpha, 1\rangle=\langle b, \alpha\rangle=\int_{b} \alpha,
$$

for elements $\alpha=D^{-1} a$ of $H^{*}(N, N \backslash X)$. In particular, when $X=N$ is a compact oriented manifold, then $\alpha \in \check{\mathrm{H}}_{c}^{*}(N)$ is Poincaré dual to $a$ under (A.23) (cf. [Br2, V.11]).

Example A.4. Let $f$ and $g$ be proper maps as in Lemma A.3, and assume that the intersection of their images are compact. Then (A.32) is a compact 0-dimensional oriented manifold, consisting of finitely many points $x$ with sign $\varepsilon(x)= \pm 1$. In this case, using (A.33), the intersection number is

$$
f_{*}[M] \cdot g_{*}[P]=\varepsilon\left(f_{*}[M] \bullet g_{*}[P]\right)=\varepsilon\left(h_{*}[Z]\right)=\sum_{x \in Z} \varepsilon(x) \in \mathbb{Z} .
$$

\section{Appendix B. Dimension theory}

One can define the dimension of a topological space $X$ in several ways:

(1) The Lebesgue covering dimension $\operatorname{dim} X$ is the smallest number $d$ so that every open cover has a refinement such that every $x \in X$ lies in at most $d+1$ sets of the refinement.

(2) The (large) cohomological dimension $\operatorname{dim}_{2} X$ is the largest $k$ such that $\check{\mathrm{H}}^{k}(X, A ; \mathbb{Z})$ is non-zero for some closed set $A$ in $X$.

In addition, if $X$ is a metric space, one has:

(3) The Hausdorff dimension $\operatorname{dim}_{H} X$ of $\mathrm{X}$ is the infimum of $\delta \geq 0$ with the following property: For any $\varepsilon>0, X$ can be covered by countably many sets $\left\{A_{n}\right\}$ with $\operatorname{diam}\left(A_{n}\right)<\varepsilon$ and with $\sum_{i} \operatorname{diam}\left(A_{n}\right)^{\delta}<\varepsilon$. 
Standard results of dimension theory show that

$$
\operatorname{dim}_{2} X=\operatorname{dim} X \quad \text { and } \quad \operatorname{dim} X \leq \operatorname{dim}_{H} X
$$

where the first equality holds for all non-empty paracompact Hausdorff spaces [Na, 36-15 and 37-7], and the second holds for all separable metric spaces [HW, p. 107]. These are related to condition (1.4) as follows.

Lemma B.1. If $X$ is a compact Hausdorff space with $\operatorname{dim} X=d$, then

$$
{ }^{s} \mathrm{H}_{k}(X ; \mathbb{Z})=H_{k}^{B M}(X ; \mathbb{Z})=0 \text { for all } k>d \text {. }
$$

Proof. For compact $X$ we have $\check{\mathrm{H}}^{*}(X)=\check{\mathrm{H}}_{c}^{*}(X)$. Using (B.1) and taking $A$ to be the empty set in the definition of $\operatorname{dim}_{2} X$, one sees that the condition $\operatorname{dim} X=d$ implies that $\check{\mathrm{H}}^{k}(X)=0$ for all $k>d$. Then (B.2) follows from (A.11) and the corresponding sequence for Borel-Moore homology.

Lemma B.2. Suppose that $X$ is a metric space and $M$ is a separable metrizable topological d-manifold.

(a) For any subspace $S \subseteq X$, $\operatorname{dim} S \leq \operatorname{dim} X$ and $\operatorname{dim}_{H} S \leq \operatorname{dim}_{H} X$.

(b) If $X$ is a countable union of closed subsets $X_{i}$, then $\operatorname{dim} X \leq \sup \operatorname{dim} X_{i}$ and $\operatorname{dim}_{H} X \leq$ $\sup \operatorname{dim}_{H} X_{i}$.

$$
\operatorname{dim} f(X) \leq \operatorname{dim} X
$$

(c) $\operatorname{dim} M=\operatorname{dim}_{H} M=d$.

(d) If $\bar{M}=M \cup S$ is a thin compactification of $M$ with $\operatorname{dim} S \leq d-2$, then $\operatorname{dim} \bar{M}=d$.

Proof. For Hausdorff dimension, (a), (b) and (c) follow easily from the definition. For covering dimension, (a) and (b) are Theorems 4.1.7 and 4.1.9 of [E], respectively, and (c) is Corollary 1 of Section IV.4 of [HW]. Finally, note that $M$ can be written as a countable union of closed subsets $B_{i}$ (closed balls of integer radius in some metric) each with $\operatorname{dim} B_{i}=d$. Applying (b) to $\bar{M}=S \cup \cup B_{i}$ with $\operatorname{dim} S \leq d-2$ shows that $\operatorname{dim} \bar{M}=d$.

In general, a continuous map can increase covering dimension, as occurs for Peano's spacefilling curve. One can avoid such pathologies by working with Hausdorff dimension and Lipschitz maps. Recall that a map $f: X \rightarrow Y$ between metric spaces is called Lipschitz if there is a constant $C>0$ such that

$$
\operatorname{dist}(f(x), f(y)) \leq C \operatorname{dist}(x, y)
$$

for all $x, y \in X$, and is locally Lipschitz if every point in $X$ has a neighborhood in which $f$ is Lipschitz. Note that if $f$ is locally Lipschitz then its restriction to a compact set $K \subset X$ is Lipschitz. Also note that the definition of Hausdorff dimension implies that if $f$ is Lipschitz then

$$
\operatorname{dim}_{H} f(X) \leq \operatorname{dim}_{H} X .
$$

Lemma B.3. Suppose that a subset $S$ of a metric space $Y$ is contained in the union of the images of a countable collection of maps $\varphi_{n}: U_{n} \rightarrow Y$, where each $U_{n}$ is a $\sigma$-compact topological manifold of dimension $\leq d$, and either

(i) $\varphi_{n}$ continuous and locally injective, or

(ii) $\varphi_{n}$ is locally Lipschitz, or 
(iii) $\varphi_{n}$ is a $C^{1}$ map between $C^{1}$ manifolds.

Then $\operatorname{dim} S \leq d$ and, if $S$ is compact, ${ }^{s} \mathrm{H}_{k}(S)=0$ for all $k>d$.

Proof. Each $U_{n}$ is $\sigma$-compact, so can be covered by a countable collection of open sets $\left\{B_{m n}\right\}$ with compact closures $\bar{B}_{m n}$. Then $\varphi_{n}$ restricts to maps $\varphi_{m n}: \bar{B}_{m n} \rightarrow Y$.

In case (i), $\varphi_{n}$ is locally injective, so we can assume, after refining the cover $\left\{B_{m n}\right\}$, that each $\varphi_{m n}$ is injective. Then $\varphi_{m n}$ is a continuous injection from a compact set to a Hausdorff space, therefore a homeomorphism onto its image. Since covering dimension is a homeomorphism invariant, parts (a) and (c) of Lemma B.2 show that the image satisfies

$$
\operatorname{dim} \varphi_{m n}\left(\bar{B}_{m n}\right)=\operatorname{dim} \bar{B}_{m n} \leq d .
$$

In case (ii), the assumptions also imply that $U_{n}$ is metrizable. After fixing a metric, inequalities (B.1), (B.3) and Lemma B.2(a,c) imply that

$$
\operatorname{dim} \varphi_{m n}\left(\bar{B}_{m n}\right) \leq \operatorname{dim}_{H} \varphi_{m n}\left(\bar{B}_{m n}\right) \leq \operatorname{dim}_{H} \bar{B}_{m n} \leq d .
$$

This inequality also holds in case (iii) because any $C^{1}$ map is locally Lipschitz.

In either case, we can apply parts (a) and (b) of Lemma B.2 to conclude that

$$
\operatorname{dim} S \leq \operatorname{dim} \bigcup_{m, n} \varphi_{m n}\left(\bar{B}_{m n}\right) \leq \sup _{m, n} \operatorname{dim} \varphi_{m n}\left(\bar{B}_{m n}\right) \leq d .
$$

The lemma then follows by applying Lemma B.1.

\section{REFERENCES}

[Br1] G.E. Bredon, Topology and Geometry, Springer-Verlag, 1993.

[Br2] G.E. Bredon, Sheaf Theory, second ed., McGraw-Hill, New York, 1997.

[BM] A. Borel and J.C. Moore, Homology theory for locally compact spaces, Michigan Math. J. 7 (1960), $137-160$.

[BeM] A. Beridze and L. Mdzinarishvili, On the axiomatic systems of Steenrod homology theory of compact spaces, arXiv: 1703.05070

[D] A. Dold, Lectures on algebraic topology, Springer-Verlag, Berlin, 1972.

[E] R. Engelking, Theory of Dimensions Finite and Infinite, Heldermann Verlag, Lemgo Germany, 1995.

[ES] S. Eilenberg and N.E. Steenrod, Foundations of algebraic topology, Princeton University Press, Princeton, 1952.

[F1] W. Fulton, Intersection Theory, Springer-Verlag, Berlin, 1984.

[F2] W. Fulton, Young Tableaux, Cambridge University Press, Cambridge, U.K., 1997.

[HW] W. Hurewicz and H. Wallman, Dimension theory, Princeton University Press, Princeton, N.J., 1941.

[In] H. Inassaridze, On the Steenrod homology theory of compact spaces, Michigan Math. J. 38 (1991), 323-338.

[Iv] B. Iverson, Cohomology of Sheaves, Springer-Verlag, 1986.

[IP1] E.N. Ionel and T.H. Parker, Thin compactifications and relative fundamental classes, arXiv:1512.07894.

To appear in J. Symplectic Geometry.

[Mar] S. Mardešić, Strong shape and homology, Springer-Verlag, Berlin, 2000.

[Mas] W.S. Massey, Homology and cohomology theory, Marcel Dekker, New York, 1978.

[Mas2] W.S. Massey, How to give an exposition of the Čech-Alexander-Spanier type homology theory, American Math. Monthly 85 (1978), 75-83.

[Mil] J. Milnor, On the Steenrod homology theory, Novikov Conjectures, Index Theorems and Rigidity, Vol. 1 (Oberwolfach, 1993), 79-96, London Math. Soc. Lecture Note Ser., 226, Cambridge Univ. Press, Cambridge, 1995.

[MS] D. McDuff and D. Salamon, J-holomorphic curves and symplectic topology, American Mathematical Society Colloquium Publications, 52, AMS, Providence, RI, 2004.

[Na] K. Nagami, Dimension theory, Academic Press, , New York, 1970.

[Pd] J. Pardon, An algebraic approach to virtual fundamental cycles on moduli spaces of pseudo-holomorphic curves, Geometry \& Topology 20 (2016), 779-1034. 
[RT1] Y. Ruan and G. Tian, A mathematical theory of quantum cohomology, J. Diff. Geom. 42 (1995), 259-367.

[Sk] E.G. Sklyarenko, Homology theory and the exactness axiom, Russian Math. Surveys, 24 Issue 5,, (1969), 91-142.

[Sk2] E.G. Sklyarenko, On homology theory associated with the Aleksandrov-Čech cohomology, Russian Math. Surveys, 34 Issue 6, (1979), 103-137.

[Sp1] E.H. Spanier, Algebraic Topology, McGraw-Hill, New York, 1966.

[Sp2] E.H. Spanier, Singular homology and cohomology with local coefficients and duality for manifolds, Pacific J. of Math., 160, (1993), 165-200.

[Sw] M. Schwarz, Equivalences for Morse homology, in Geometry and Topology in Dynamics, pp. 197-216, Contemporary Mathematics 246, M. Barge and K. Kuperberg, Eds,. AMS, (1999).

Stanford University, Palo Alto, CA, USA.

E-mail address: ionel@math.stanford.edu

Michigan State University, East Lansing MI, USA.

E-mail address: parker@math.msu.edu 